Article

\title{
What Every Internist-Endocrinologist Should Know about Rare Genetic Syndromes in Order to Prevent Needless Diagnostics, Missed Diagnoses and Medical Complications: Five Years of 'Internal Medicine for Rare Genetic Syndromes'
}

\author{
Anna G. W. Rosenberg 1,2 ${ }^{1}$, Minke R. A. Pater ${ }^{1}$, Karlijn Pellikaan 1,2 ${ }^{\mathbb{D}}$, Kirsten Davidse ${ }^{1,2}$, \\ Anja A. Kattentidt-Mouravieva ${ }^{3}$, Rogier Kersseboom ${ }^{3}$, Anja G. Bos-Roubos ${ }^{4}$, Agnies van Eeghen 5,6,7, \\ José M. C. Veen ${ }^{8}$, Jiske J. van der Meulen ${ }^{8}$, Nina van Aalst-van Wieringen ${ }^{9}$, Franciska M. E. Hoekstra ${ }^{1,10}$, \\ Aart J. van der Lely ${ }^{1,+} \mathbb{D}$ and Laura C. G. de Graaff $1,2,7,11,12,13, *,+$
}

Citation: Rosenberg, A.G.W.; Pater M.R.A.; Pellikaan, K.; Davidse, K.; Kattentidt-Mouravieva, A.A.; Kersseboom, R.; Bos-Roubos, A.G.; van Eeghen, A.; Veen, J.M.C.; van der Meulen, J.J.; et al. What Every Internist-Endocrinologist Should Know about Rare Genetic Syndromes in Order to Prevent Needless Diagnostics, Missed Diagnoses and Medical Complications: Five Years of 'Internal Medicine for Rare Genetic Syndromes'. J. Clin. Med. 2021, 10, 5457. https://doi.org/10.3390/ jcm10225457

Academic Editors: Assumpta Caixàs and Gianluca Castelnuovo

Received: 25 October 2021

Accepted: 17 November 2021

Published: 22 November 2021

Publisher's Note: MDPI stays neutral with regard to jurisdictional claims in published maps and institutional affiliations.

Copyright: (c) 2021 by the authors. Licensee MDPI, Basel, Switzerland. This article is an open access article distributed under the terms and conditions of the Creative Commons Attribution (CC BY) license (https:/ / creativecommons.org/licenses/by/ $4.0 /)$.
1 Department of Internal Medicine, Division of Endocrinology, Erasmus MC, University Medical Center Rotterdam, 3015 GD Rotterdam, The Netherlands; a.rosenberg@erasmusmc.nl (A.G.W.R.); m.pater@erasmusmc.nl (M.R.A.P.); k.pellikaan@erasmusmc.nl (K.P.); k.davidse@erasmusmc.nl (K.D.); f.hoekstra@rdgg.nl (F.M.E.H.); a.vanderlelij@erasmusmc.nl (A.J.v.d.L.)

2 Dutch Center of Reference for Prader-Willi Syndrome, 3015 GD Rotterdam, The Netherlands

3 Stichting Zuidwester, 3241 LB Middelharnis, The Netherlands; a.kattentidt@zuidwester.org (A.A.K.-M.); r.kersseboom@zuidwester.org (R.K.)

4 Center of Excellence for Neuropsychiatry, Vincent van Gogh, 5803 DN Venray, The Netherlands; aroubos@vvgi.nl

5 's Heeren Loo, Care Group, 3818 LA Amersfoort, The Netherlands; a.m.vaneeghen@amsterdamumc.nl

6 Department of Pediatrics, Amsterdam University Medical Center, 1105 AZ Amsterdam, The Netherlands

7 Academic Center for Growth Disorders, Erasmus MC, University Medical Center Rotterdam, 3015 GD Rotterdam, The Netherlands

8 's Heeren Loo, Care Providing Agency, 6733 SC Wekerom, The Netherlands; josedietist@gmail.com (J.M.C.V.); jiske.van-der-meulen@sheerenloo.nl (J.J.v.d.M.)

9 Department of Physical Therapy, Erasmus MC, University Medical Center Rotterdam, 3015 GD Rotterdam, The Netherlands; n.vanaalst-vanwieringen@erasmusmc.nl

10 Department of Internal Medicine, Reinier de Graaf Hospital, 2625 AD Delft, The Netherlands

11 ENCORE-Dutch Center of Reference for Neurodevelopmental Disorders, 3015 GD Rotterdam, The Netherlands

12 Dutch Center of Reference for Turner Syndrome, 3015 GD Rotterdam, The Netherlands

13 Dutch Center of Reference for Disorders of Sex Development, 3015 GD Rotterdam, The Netherlands

* Correspondence: 1.degraaff@erasmusmc.nl

+ ENDO-ERN, European Reference Network on Rare Endocrine Conditions.

Abstract: Patients with complex rare genetic syndromes (CRGS) have combined medical problems affecting multiple organ systems. Pediatric multidisciplinary (MD) care has improved life expectancy, however, transfer to internal medicine is hindered by the lack of adequate MD care for adults. We have launched an MD outpatient clinic providing syndrome-specific care for adults with CRGS, which, to our knowledge, is the first one worldwide in the field of internal medicine. Between 2015 and 2020, we have treated 720 adults with over 60 syndromes. Eighty-nine percent of the syndromes were associated with endocrine problems. We describe case series of missed diagnoses and patients who had undergone extensive diagnostic testing for symptoms that could actually be explained by their syndrome. Based on our experiences and review of the literature, we provide an algorithm for the clinical approach of health problems in CRGS adults. We conclude that missed diagnoses and needless invasive tests seem common in CRGS adults. Due to the increased life expectancy, an increasing number of patients with CRGS will transfer to adult endocrinology. Internist-endocrinologists (in training) should be aware of their special needs and medical pitfalls of CRGS will help prevent the burden of unnecessary diagnostics and under- and overtreatment.

Keywords: syndrome; intellectual disability; missed diagnosis; medical overuse; internal medicine; multidisciplinary care 


\section{Introduction}

Patients with complex genetic syndromes (CRGS), by definition, have combined medical problems affecting multiple organ systems [1-3]. Intellectual disability and challenging behavior are often part of the syndrome. During the last decades, healthcare for children with CRGS has significantly improved. Most children with CRGS receive multidisciplinary (MD) and specialized medical care in which usually three to four medical specialists are involved [4]. Whereas in the past, many genetic syndromes were associated with premature death, this improvement of medical care has increased life expectancy and a growing number of children with CRGS now reach adult age [5,6]. Although the complexity of the manifestations of CRGS generally persists into adulthood, proper syndrome-specific MD hospital care is rarely available for adults with CRGS. While multiple organ systems are usually affected, specialists with a broad scope, like internists and endocrinologists, are seldomly involved. After transfer from pediatric to adult medical care, patients and their parents often report fragmented care of poor quality instead of adequate and integrated health management [7-15]. Furthermore, information on the natural course and medical guidelines are often lacking for these 'new adults'. In our experience, the lack of adequate information can lead to undiagnosed health problems, suboptimal treatment, and painful and expensive complications. Therefore, pediatricians and intellectual disability physicians (ID physicians) have expressed the urgent need for adequate, specialized, MD ternary hospital care for adults with CRGS $[16,17]$. To address this need, we have launched a specialized MD outpatients' clinic (MOPC) within the Department of Internal Medicine, Division of Endocrinology, specially designed for adults with CRGS. The MD team consists of an internist-endocrinologist, neuropsychologist, ID physician, clinical geneticist, dietitian for intellectual disability, physiotherapist, and nurse practitioner. As MD care for adults with CRGS is not available elsewhere in the Netherlands, our center serves as a ternary referral center. This results in the clustering of relatively large numbers of patients with rare syndromes in our center, which gives us the unique opportunity to study their medical needs and syndrome-specific manifestations. In this article, we describe our experience of the first five years of 'Internal Medicine-Endocrinology for Complex Rare Genetic Syndromes'. We report the most frequent syndrome-specific complaints and provide an illustrative case series of patients who underwent unnecessary (invasive) diagnostics tests for symptoms and complaints that were either direct (primary somatic manifestations) or indirect consequences of the syndrome. Based on our experience and previous literature, we provide an overview of syndrome-specific endocrine and non-endocrine manifestations and provide practical advice for diagnostics and treatment. By sharing our experience, we aim to show the importance of specialized MD care for adults with CRGS. Our data will help other internist-endocrinologists prepare for the increasing numbers of patients with CRGS that are now reaching adult age and transferring to internal medicine/adult endocrinology. Creating awareness of the special needs of adults with CRGS and of the pitfalls in diagnosis and treatment will likely prevent unnecessary interventions and prevent painful and expensive complications in this vulnerable patient group.

\section{Materials and Methods}

Approval for this retrospective study was waived by the local Medical Ethics Committee of the Erasmus University Medical Center Rotterdam (EMCR, Rotterdam, The Netherlands). Informed consent from individual patients or their legal representatives was obtained for the case series. We reviewed the medical files of all adults who visited the Center for CRGS at the Endocrinology Unit of the Department of Internal Medicine of the EMCR between April 2015 and December 2020. Although Klinefelter syndrome (KS) does not meet the European definition of rare diseases (1:2000 persons affected [18]), $\mathrm{KS}$ is included in this article as adults with KS also visited our center for CRGS. As adult manifestations are unknown for most CRGS and as history taking may be hindered by ID, pre-visit medical questionnaires and systematic health screening are standard procedures of routine patient care of our center. The systematic screening consists of a medical 
questionnaire, structured interview (including use of medication), a complete physical examination, a review of the medical records, biochemical measurements, and, if indicated and feasible, additional tests. Part of the data on Prader-Willi syndrome (PWS) was previously published [19].

\subsection{Genetic Diagnosis}

Genetic confirmation was either collected from the referring physician or was performed at our medical center to confirm the clinical diagnosis.

\subsection{Medical Questionnaire}

As part of regular patient care, patients or their primary caregivers filled out a questionnaire before visiting the MOPC. The questionnaire included items on the patient's medical history, medication, family history, symptoms of disease, physical complaints, behavioral challenges, and social aspects (work, school, relationship, and living situation). The items "symptoms of disease", "physical complaints", and "behavioral challenges" were rated on a 5-point Likert scale ( $1=$ rarely or never, $2=$ not often and /or not severe, $3=$ quite often and/or quite severe, $4=$ often and/or severe, $5=$ very often and/or very severe). A score of 3 or higher was considered clinically relevant and was further examined during the visit. The composition of the MD team was based on the answers that were provided in the pre-visit questionnaire.

\subsection{Biochemical Analysis}

During the visit, blood samples were taken for general screening, including evaluation of the hematopoietic system, kidney function, liver enzymes, glucose metabolism, fat metabolism, thyroid function, gonadal function, and vitamin D status.

\subsection{Additional Tests}

We screened for hypertension at the first visit to our MOPC. In case of a blood pressure above $140 / 90 \mathrm{mmHg}$, the measurement was repeated and if it was still elevated, a $30 \mathrm{~min}$ blood pressure measurement was performed. Patients were diagnosed with borderline hypertension if the first blood pressure measurement was above $140 / 90 \mathrm{mmHg}$ and the repeated blood pressure measurements were around 140/90 $\mathrm{mmHg}$. Other additional tests that we often perform at our center, but are beyond the scope of this article, include dual-energy X-ray absorptiometry in case of clinical suspicion of osteoporosis or osteopenia and polygraphy or polysomnography in case of clinical suspicion of sleep apnea.

\subsection{Literature Search}

Literature was searched in several databases (MEDLINE (via ALL OVID), Embase, and Cochrane Central Register of Controlled Trials) for clinical manifestations of the syndromes that we encountered at our MOPC. The full search strategy is available upon request.

\subsection{Data Analysis}

Data were analyzed with IBM SPSS version 25 and R version 3.6.0. Data are presented as the mean \pm standard deviation for continuous data and percentage (number) for categorical data. We clustered "daytime sleepiness" and "general muscle weakness" with "tiredness", as in our experience, patients use the term "tiredness" also to express "daytime sleepiness" and "general (muscle) weakness". The term "fatigue" represents the clustered variable. "Abdominal complaints" were defined as the presence of constipation, diarrhea, and/or abdominal pain. Frequencies of complaints are reported separately for the syndromes for which syndrome-specific questionnaires were available, namely Prader-Willi syndrome (PWS), Neurofibromatosis type 1 (NF1), KS, and "other CRGS". 


\section{Results}

During the first 5 years of the MOPC, we have treated 720 adults, with 61 different CRGS (Table 1). In 41 patients, there was a clinical suspicion of a genetic syndrome (due to the combination of dysmorphic features, intellectual disabilities, and/or dysfunction of multiple organs) but the underlying genetic etiology was unknown.

Table 1. Overview of complex rare genetic syndromes seen in our multidisciplinary outpatient clinic.

\begin{tabular}{|c|c|c|c|c|c|}
\hline Syndrome & $\mathbf{N}$ & Syndrome & $\mathbf{N}$ & Syndrome & $\mathbf{N}$ \\
\hline Albright hereditary osteodystrophy & $<5$ & Dandy-Walker syndrome & $<5$ & Ring chromosome 21 & $<5$ \\
\hline Allan-Herndon-Dudley syndrome & $<5$ & $\begin{array}{l}\text { DiGeorge syndrome } \\
\text { (22q11.2 deletion) }\end{array}$ & 8 & Saethre-Chotzen syndrome & $<5$ \\
\hline Alström syndrome & $<5$ & $\begin{array}{l}\text { Disorders of Sex } \\
\text { Development }^{1}\end{array}$ & 18 & $\begin{array}{l}\text { Say-Barber-Biesecker-Young- } \\
\text { Simpson syndrome } \\
\text { (KAT6B mutation) }\end{array}$ & $<5$ \\
\hline Angelman syndrome & $<5$ & $\begin{array}{l}\text { Down syndrome } \\
\text { (trisomy } 21)\end{array}$ & $<5$ & $\begin{array}{l}\text { Sifrim-Hitz-Weiss } \\
\text { syndrome }\end{array}$ & $<5$ \\
\hline Axenfeld-Rieger syndrome & $<5$ & $\begin{array}{c}\text { Hypogonadotropic } \\
\text { hypogonadism with anosmia } \\
\text { (Kallmann syndrome) }\end{array}$ & 6 & Silver-Russell syndrome & 5 \\
\hline Bardet-Biedl syndrome & 5 & $\begin{array}{l}\text { Hypogonadotropic } \\
\text { hypogonadism without anosmia } \\
\text { (Kiss1R mutation) }\end{array}$ & $<5$ & $\begin{array}{l}\text { Smith-Lemli-Opitz } \\
\text { syndrome }\end{array}$ & $<5$ \\
\hline Bloom syndrome & $<5$ & Jacobsen syndrome & $<5$ & Smith-Magenis syndrome & $<5$ \\
\hline $\begin{array}{l}\text { Börjeson-Forssman-Lehmann } \\
\text { syndrome }\end{array}$ & $<5$ & Joubert syndrome & $<5$ & Sotos-like syndrome & $<5$ \\
\hline CAMK2A variants & $<5$ & JS-X syndrome & $<5$ & $\begin{array}{c}\text { Tatton-Brown-Rahman } \\
\text { syndrome }\end{array}$ & $<5$ \\
\hline CHARGE syndrome & 10 & Kabuki syndrome & $<5$ & TBL1X mutation & $<5$ \\
\hline CHD8 syndrome & $<5$ & KAT6A syndrome & $<5$ & Tetra- $X$ syndrome & $<5$ \\
\hline Chromosome 1q21 deletion syndrome & $<5$ & Klinefelter syndrome & 41 & Triple-X syndrome & $<5$ \\
\hline Chromosome 1q25-32 deletion & $<5$ & L1CAM mutation & $<5$ & TRPV4 mutation & $<5$ \\
\hline $\begin{array}{c}\text { Chromosome } 16 \mathrm{p} 11.2 \text { deletion } \\
\text { syndrome }\end{array}$ & $<5$ & Myhre syndrome & $<5$ & Tuberous sclerosis complex & 49 \\
\hline $\begin{array}{c}\text { Chromosome } 16 \mathrm{p} 3.11 \text { deletion } \\
\text { syndrome }\end{array}$ & $<5$ & Neurofibromatosis type 1 & 120 & Turner syndrome & 184 \\
\hline Cockayne syndrome & $<5$ & Noonan syndrome & 9 & Williams-Beuren syndrome & 10 \\
\hline Congenital adrenal hyperplasia & 11 & PNPLA6 gene mutation & $<5$ & $\begin{array}{c}\text { 45,X/46,XY mixed gonadal } \\
\text { dysgenesis }\end{array}$ & $<5$ \\
\hline Cornelia de Lange syndrome & $<5$ & $\begin{array}{l}\text { PTEN hamartoma tumor } \\
\text { syndrome }\end{array}$ & $<5$ & $48, X X X Y$ syndrome & $<5$ \\
\hline Costello (like) syndrome & $<5$ & Prader-Willi like syndrome & 9 & $48, X X Y Y$ syndrome & $<5$ \\
\hline Cri-du-chat syndrome & $<5$ & Prader-Willi syndrome & 135 & Unknown syndrome & 41 \\
\hline CTNNB1 syndrome & $<5$ & Rett syndrome & $<5$ & Total & 720 \\
\hline
\end{tabular}

Abbreviations: N, number of patients. As some syndromes only occurred in a very small number of patients, a threshold of 5 was chosen for the sake of patient privacy. ${ }^{1}$ This includes complete androgen insensitivity syndrome, Leydig cell hypoplasia, partial androgen insensitivity syndrome, $5 \alpha$-reductase deficiency, $17 \beta$-hydroxysteroid dehydrogenase type 3 deficiency, 17-20 desmolase deficiency, and partial 17-hydroxylase deficiency, and 46,XX + SRY. The bold distinguishes the total number of patients clearly from the syndromes.

Apart from 41 men with KS and 184 women with Turner syndrome, $43 \%(\mathrm{~N}=211)$ of our patient population were male and $57 \%(\mathrm{~N}=284)$ were female. The mean $\pm \mathrm{SD}$ age was $35.8 \pm 13.3$ years. The medical questionnaire was filled out by 369 patients of which 100 PWS patients, 97 NF1 patients, 29 KS patients, and 143 patients with other genetic CRGS. Figures 1-4 show the physical complaints reported by the patient and/or their primary caregivers in the medical questionnaire. The most frequent complaints were fatigue $(60 \%$; $\mathrm{N}=223$ ) and abdominal complaints (37\%; $\mathrm{N}=135)$; the prevalence of these complaints differed between the different CRGS (Figures 1-4). The population was characterized by polypharmacy and the most frequently used drugs were psychotropic drugs $(22 \% ; \mathrm{N}=56$ out 251$)$ and pain medication ( $22 \%, \mathrm{~N}=25$ out 112$)$. 


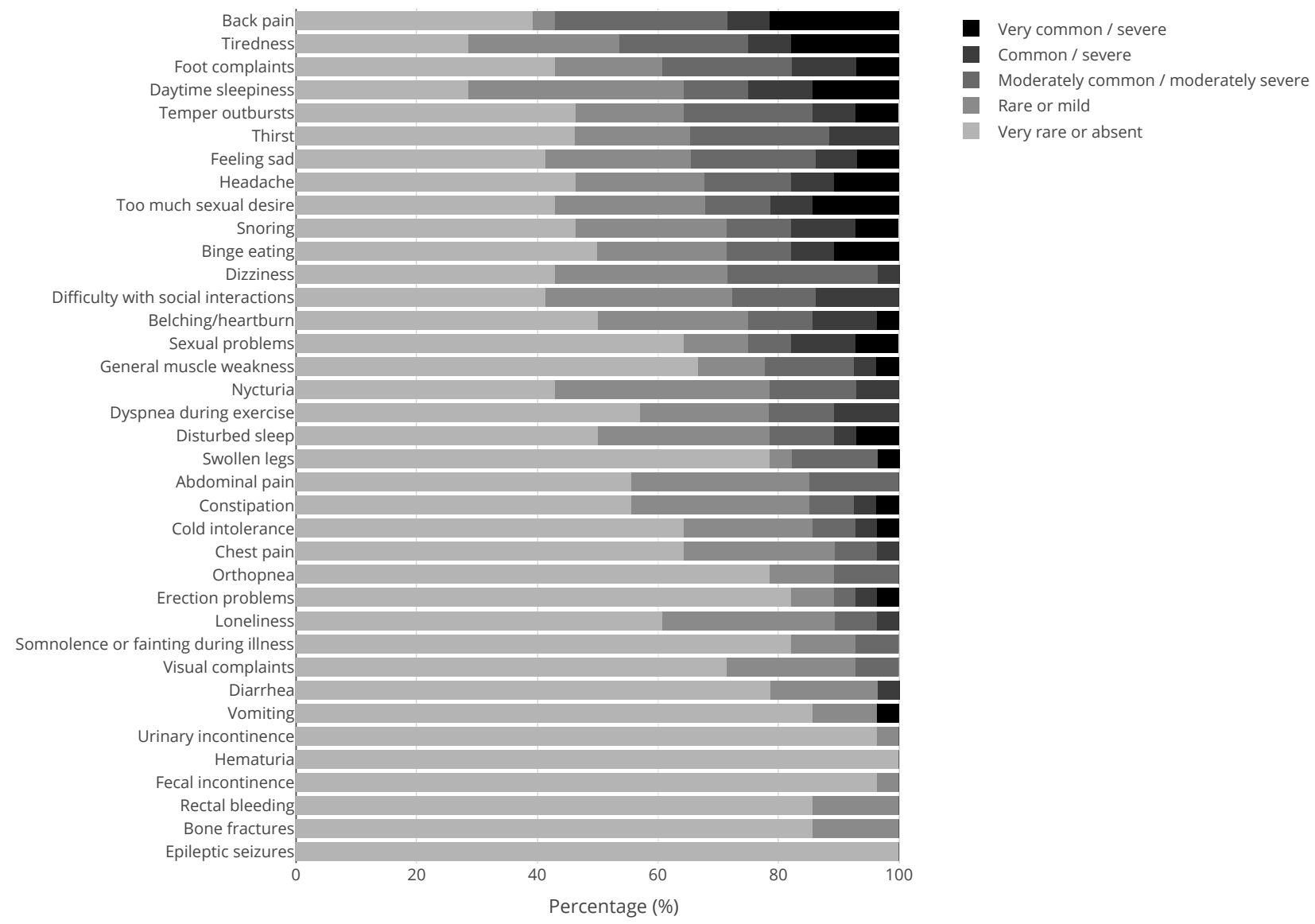

Figure 1. Physical complaints reported by (caregivers of) adults with Klinefelter syndrome $(\mathrm{N}=29)$. The tones of grey represent the scores 1 (rarely or never; lightest shade) to 5 (very often and/or very severe; darkest shade), see also the Section 2 . The dree darkest tones of grey are considered clinically relevant (score $\geq 3$ ).
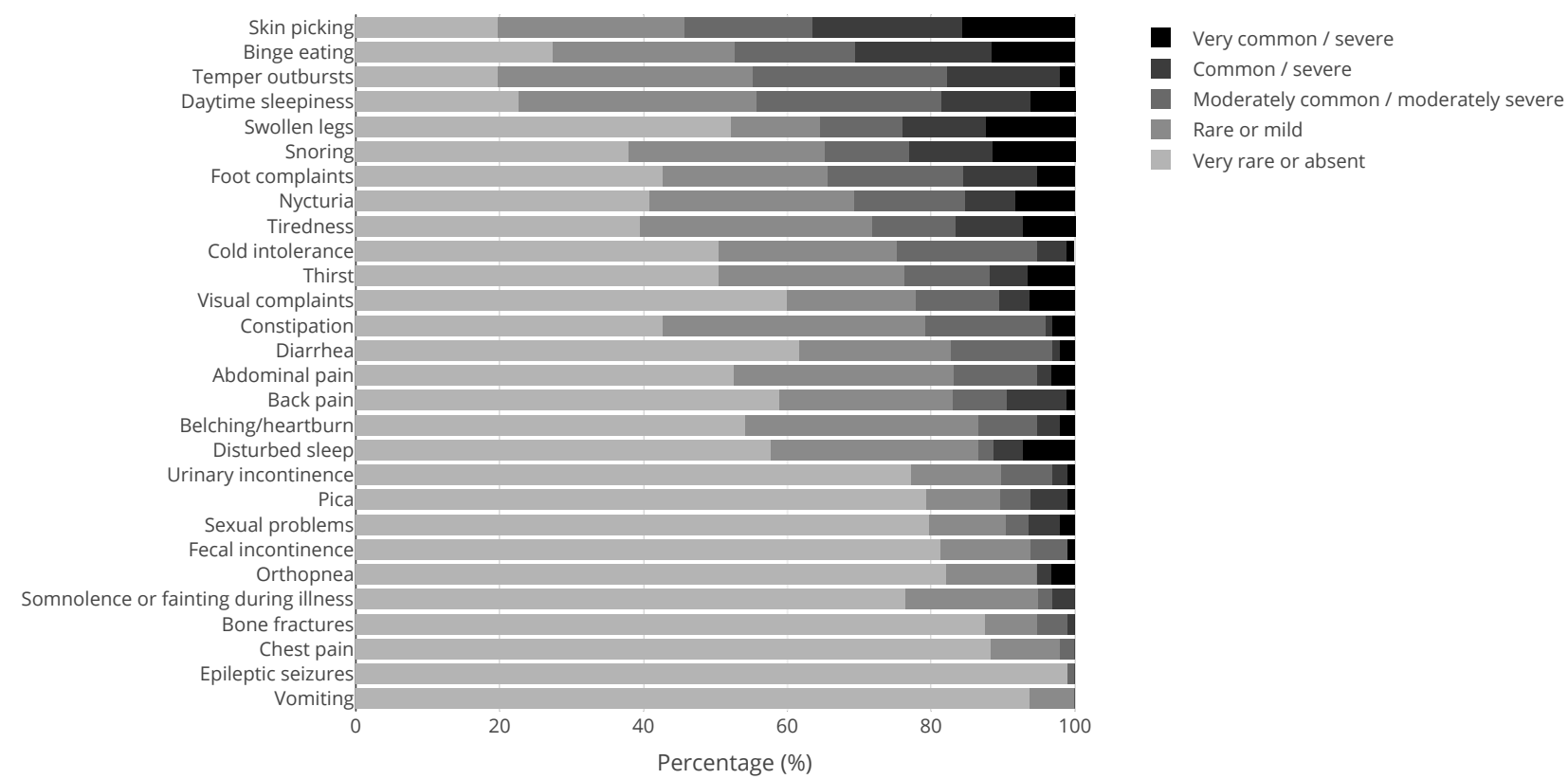

Figure 2. Physical complaints reported by (caregivers of) adults with Prader-Willi syndrome $(\mathrm{N}=100)$. The tones of grey represent the scores 1 (rarely or never; lightest shade) to 5 (very often and/or very severe; darkest shade), see also the Section 2 . The dree darkest tones of grey are considered clinically relevant (score $\geq 3$ ). 


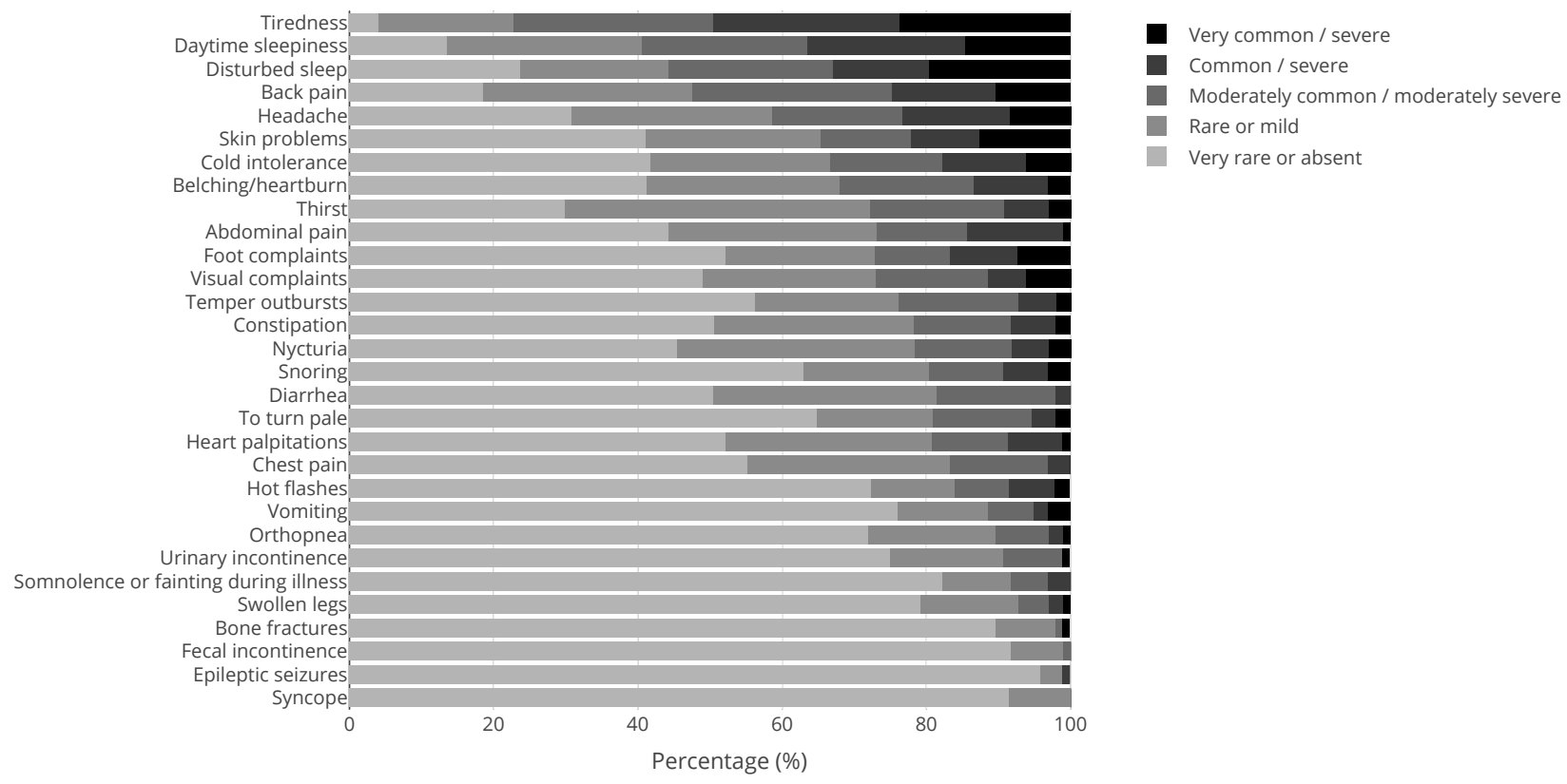

Figure 3. Physical complaints reported by (caregivers of) adults with Neurofibromatosis type 1 ( $\mathrm{N}=97$ ). The tones of grey represent the scores 1 (rarely or never; lightest shade) to 5 (very often and/or very severe; darkest shade), see also the Section 2. The dree darkest tones of grey are considered clinically relevant (score $\geq 3$ ).
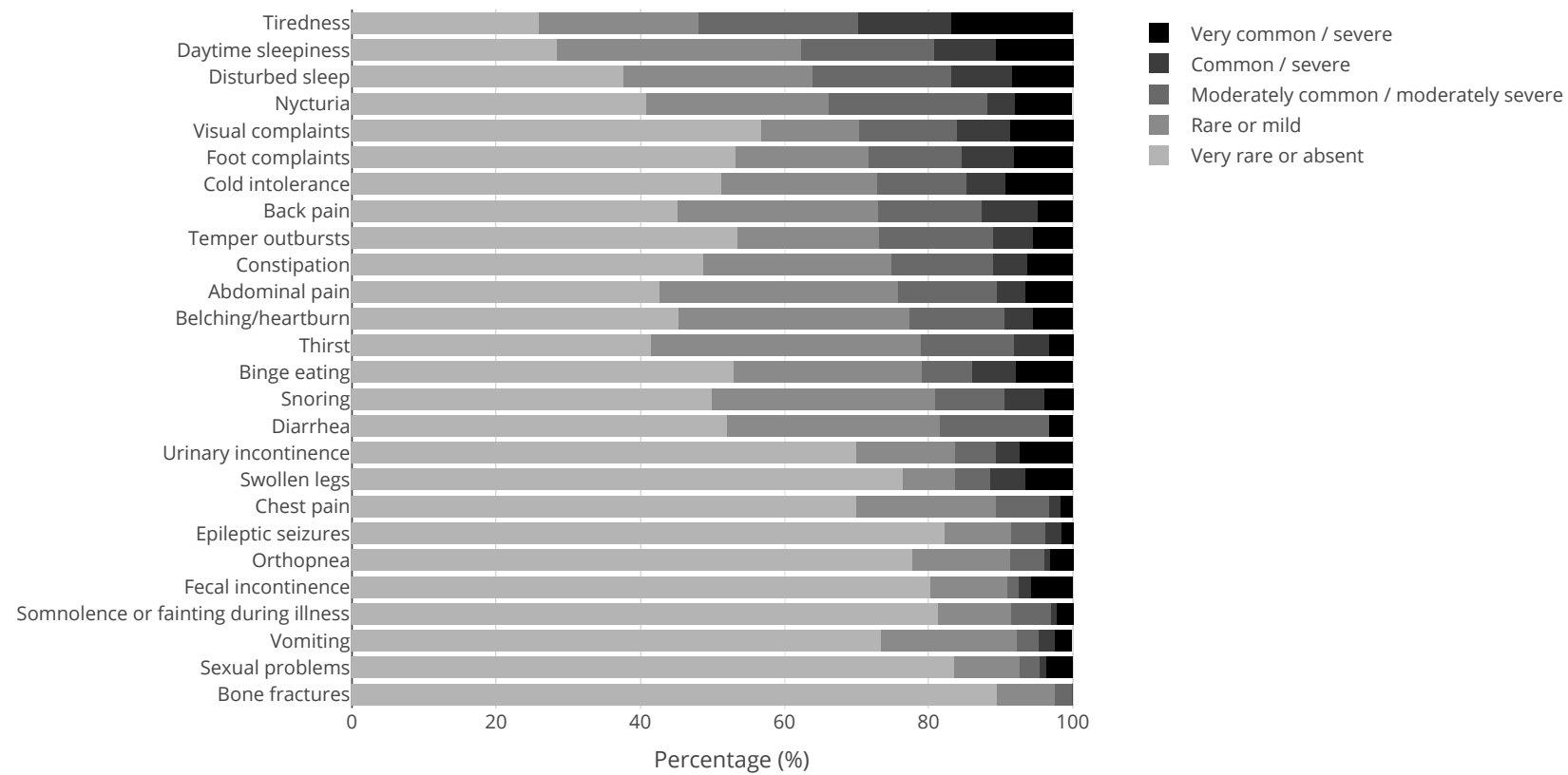

Figure 4. Physical complaints reported by (caregivers of) adults with rare genetic syndromes other than Prader-Willi syndrome, Neurofibromatosis type, or Klinefelter syndrome $(\mathrm{N}=143)$. The tones of grey represent the scores 1 (rarely or never; lightest shade) to 5 (very often and/or very severe; darkest shade), see also the Section 2 . The dree darkest tones of grey are considered clinically relevant (score $\geq 3$ ).

Table 2 shows the clinical manifestations of all CRGS seen in our center during the study period. Eighty-nine percent of the syndromes were associated with endocrine problems and $72 \%$ with intellectual disability. High blood pressure was common; among the 720 patients who visited our center, hypertension was present in $20 \%$ ( $N=142$ out 678$)$ of the patients; $2 \%(\mathrm{~N}=11$ out 678$)$ had borderline hypertension. 
Table 2. Clinical manifestations of complex rare genetic disorders seen in our center since 2015.

\begin{tabular}{|c|c|c|c|}
\hline & Endocrine Manifestations & Internal Medicine-Other & Other Disciplines \\
\hline $\begin{array}{l}\text { Albright hereditary osteodystrophy } \\
\qquad[20,21]\end{array}$ & 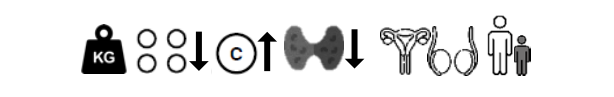 & 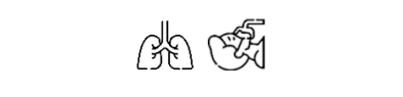 & 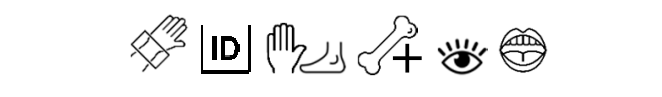 \\
\hline $\begin{array}{c}\text { Allan-Herndon- } \\
\text { Dudley syndrome } \\
{[22,23]}\end{array}$ & 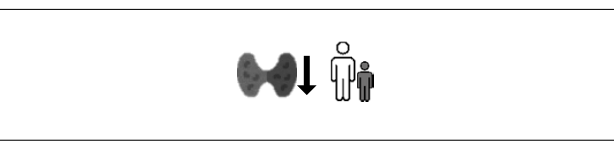 & 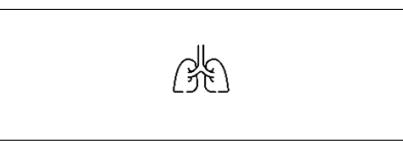 & 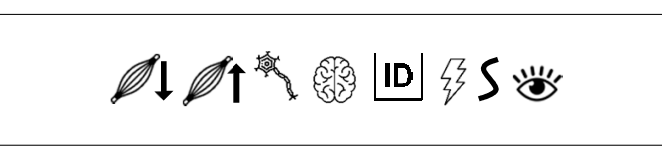 \\
\hline $\begin{array}{c}\text { Alström syndrome } \\
{[24,25]}\end{array}$ & 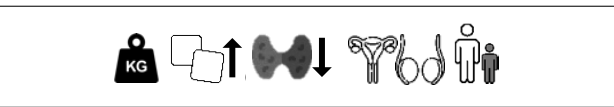 & 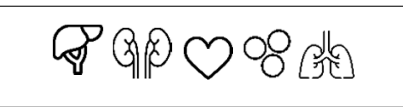 & 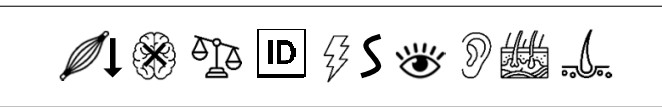 \\
\hline Angelman syndrome $[26,27]$ & 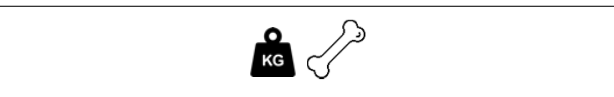 & 成数 & 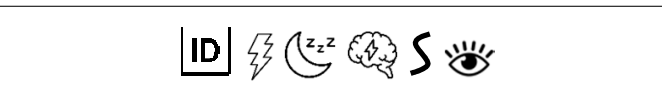 \\
\hline $\begin{array}{l}\text { Axenfeld-Rieger } \\
\text { syndrome }[28,29]\end{array}$ & & $M P O$ & 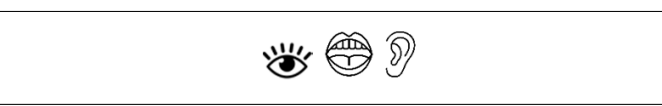 \\
\hline $\begin{array}{l}\text { Bardet-Biedl } \\
\text { syndrome [30-34] }\end{array}$ & Кำ & 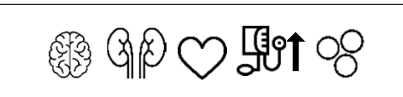 & 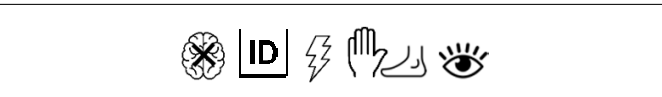 \\
\hline $\begin{array}{l}\text { Bloom syndrome } \\
{[35]}\end{array}$ & 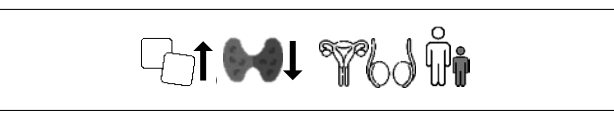 & 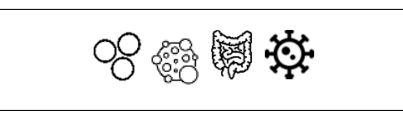 & ID \\
\hline $\begin{array}{l}\text { Börjeson-Forssman-Lehmann syndrome } \\
{[36,37]}\end{array}$ & 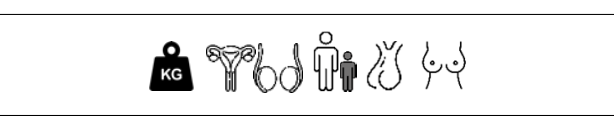 & & 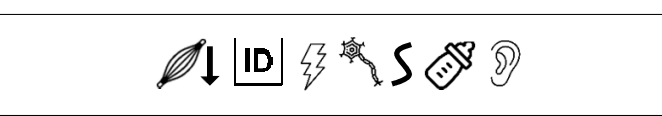 \\
\hline $\begin{array}{c}\text { CAMK2A variants } \\
{[38,39]}\end{array}$ & 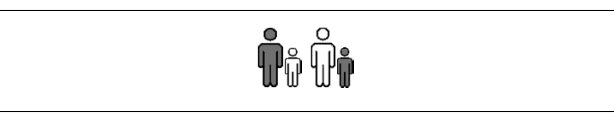 & 0 & 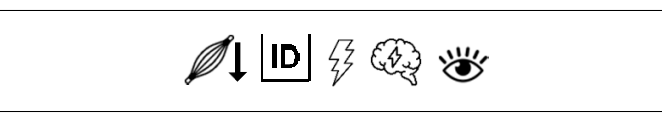 \\
\hline $\begin{array}{l}\text { CHARGE syndrome } \\
\text { [40-51] }\end{array}$ & 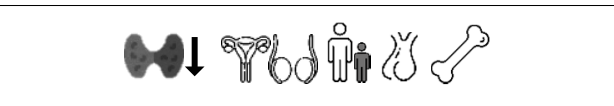 & 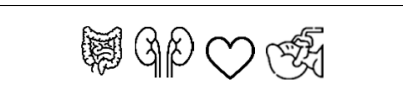 & 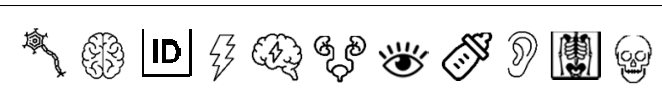 \\
\hline $\begin{array}{l}\text { CHD8 syndrome } \\
{[52-54]}\end{array}$ & 踶 & 堣 & 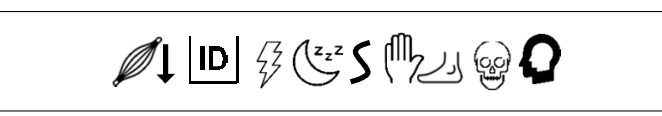 \\
\hline $\begin{array}{c}\text { Chromosome 1q21 } \\
\text { deletion syndrome [55-57] }\end{array}$ & 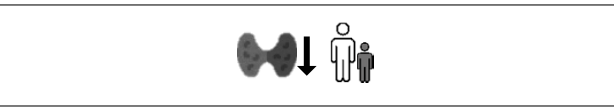 & $\mathrm{O}$ & 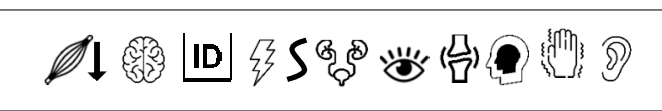 \\
\hline Chromosome 1q25-32 deletion [58,59] & OI 迎 & & ID \\
\hline
\end{tabular}


Table 2. Cont.

\begin{tabular}{|c|c|c|c|}
\hline & Endocrine Manifestations & Internal Medicine-Other & Other Disciplines \\
\hline $\begin{array}{l}\text { Chromosome 16p11.2 deletion syndrome } \\
\text { [60-62] }\end{array}$ & кө & 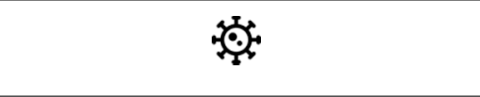 & 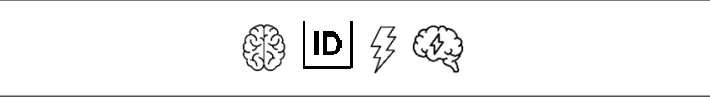 \\
\hline $\begin{array}{l}\text { Chromosome 16p13.11 deletion syndrome } \\
\text { [63-65] }\end{array}$ & & 閣 & 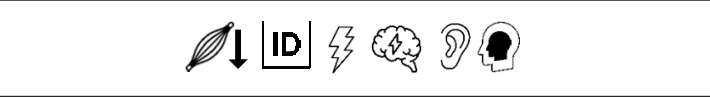 \\
\hline Cockayne syndrome [66-70] & 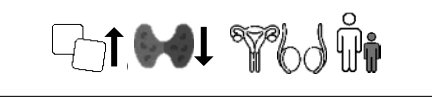 & 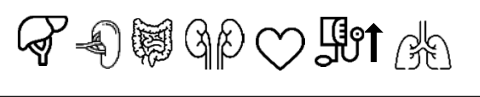 & 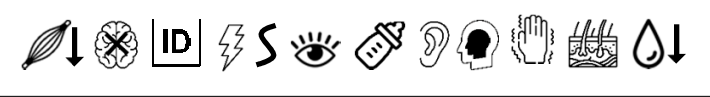 \\
\hline $\begin{array}{l}\text { Congenital adrenal } \\
\text { hyperplasia [71,72] }\end{array}$ & 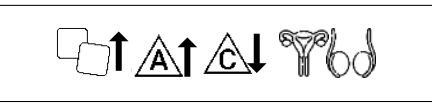 & 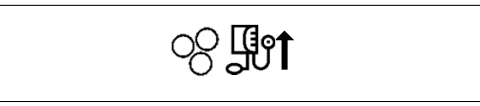 & Q \\
\hline Cornelia de Lange syndrome [73-77] & 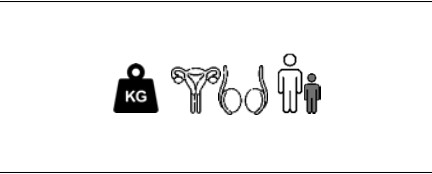 & 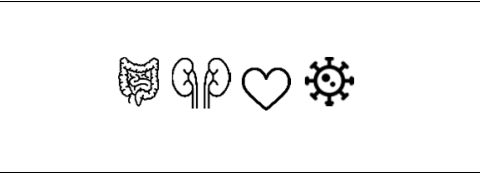 & 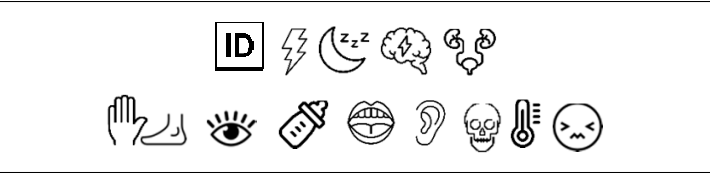 \\
\hline $\begin{array}{l}\text { Costello (like) } \\
\text { syndrome [78-80] }\end{array}$ & 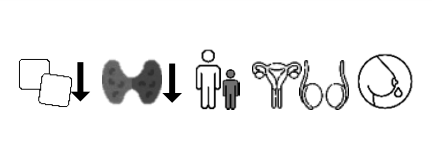 & 成 & 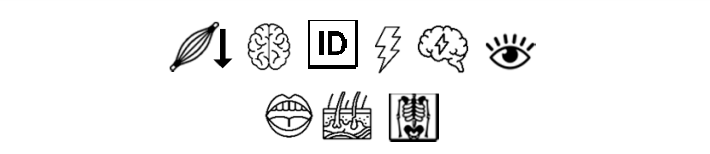 \\
\hline Cri-du-chat syndrome [81-84] & & g) & 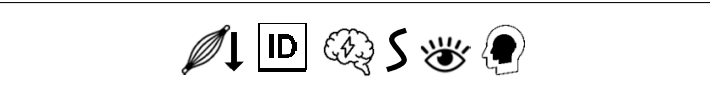 \\
\hline $\begin{array}{l}\text { CTNNB1 syndrome (NEDSDV syndrome) } \\
\text { [85-87] }\end{array}$ & & 解 & 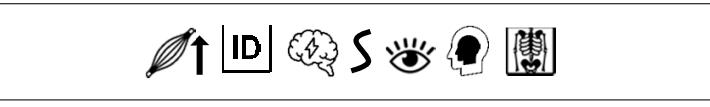 \\
\hline $\begin{array}{l}\text { Dandy-Walker } \\
\text { syndrome [88,89] }\end{array}$ & 品魚 & G/PO & 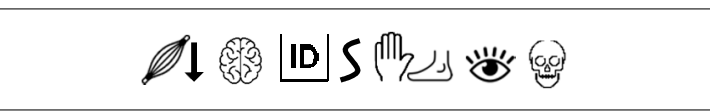 \\
\hline $\begin{array}{l}\text { DiGeorge syndrome (22q11.2 deletion) } \\
\qquad[90-105]\end{array}$ & 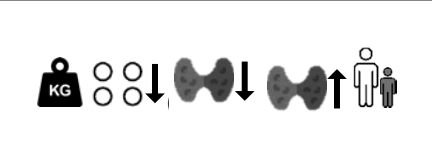 & 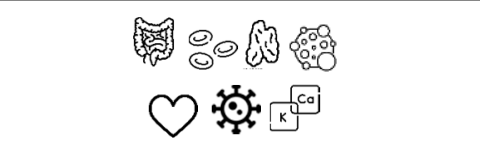 & 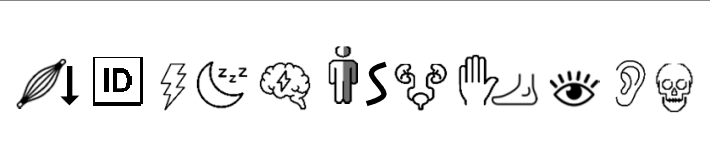 \\
\hline $\begin{array}{c}\text { Disorders of Sex } \\
\text { Development } t^{1}[106,107]\end{array}$ & $c 1$ pibd & 장 & $\mathrm{S}_{3}$ \\
\hline $\begin{array}{l}\text { Down syndrome } \\
\text { (trisomy 21) [108-113] }\end{array}$ & 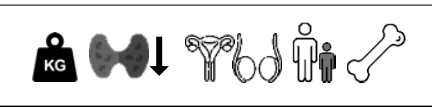 & 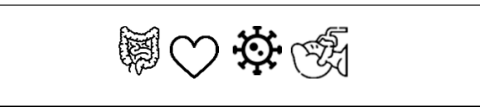 & 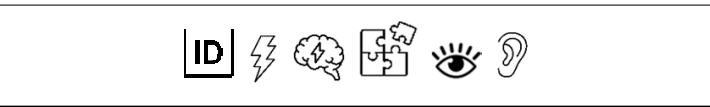 \\
\hline
\end{tabular}


Table 2. Cont.

\begin{tabular}{|c|c|c|c|}
\hline & Endocrine Manifestations & Internal Medicine-Other & Other Disciplines \\
\hline $\begin{array}{c}\text { Hypogonadotropic } \\
\text { hypogonadism with anosmia (Kallmann } \\
\text { syndrome) [114,115] }\end{array}$ & 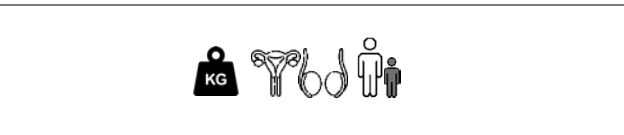 & $9, p$ & 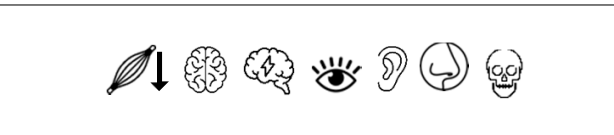 \\
\hline $\begin{array}{c}\text { Hypogonadotropic } \\
\text { hypogonadism } \\
\text { without anosmia } \\
\text { (Kiss 1R mutation) [116,117] }\end{array}$ & 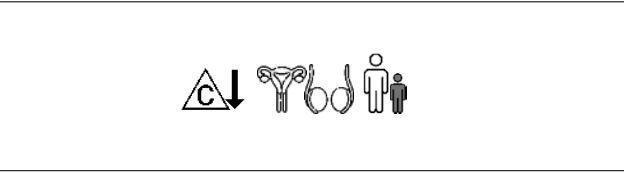 & & \\
\hline $\begin{array}{l}\text { Jacobsen syndrome (11q terminal deletion } \\
\text { syndrome) [118-121] }\end{array}$ & 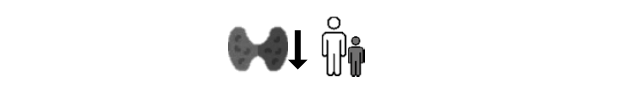 & 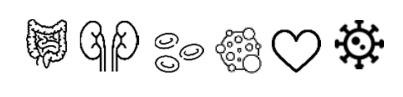 & 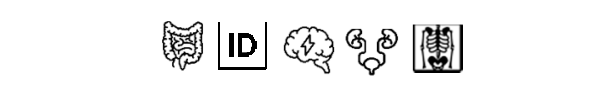 \\
\hline Joubert syndrome $[122,123]$ & 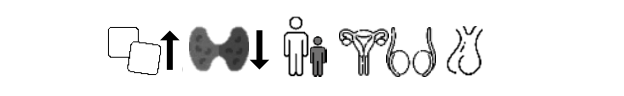 & 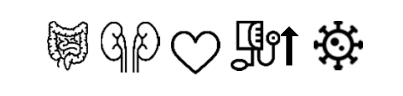 & 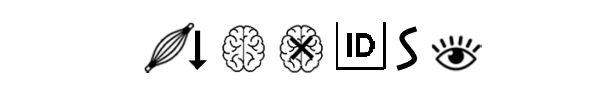 \\
\hline JS-X syndrome [124] & & & 问句器 \\
\hline Kabuki syndrome [125-129] & Ko & 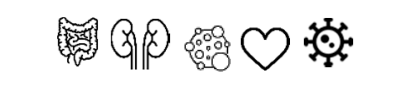 & 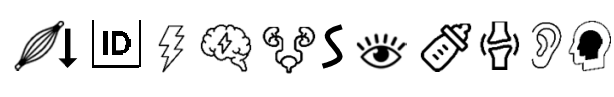 \\
\hline KAT6A syndrome $[130,131]$ & pibd & ○察 & 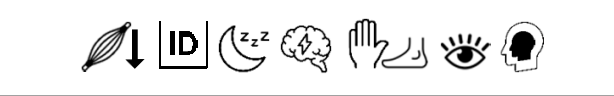 \\
\hline Klinefelter syndrome [132-138] & 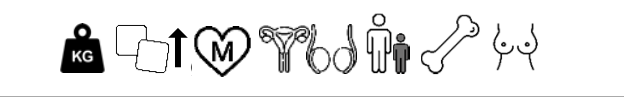 & O৩ & Q \\
\hline L1CAM mutation [139-141] & 品鱼 & 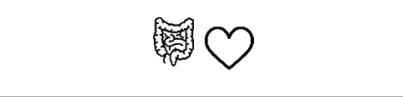 & 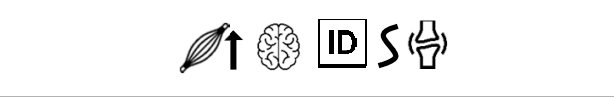 \\
\hline $\begin{array}{c}\text { Myhre syndrome } \\
\text { [142-144] }\end{array}$ & 品 97 & 县9个 & 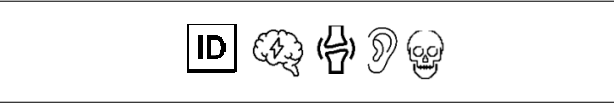 \\
\hline Neurofibromatosis type 1 [145-154] & ผ & 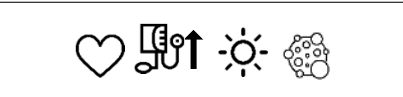 & 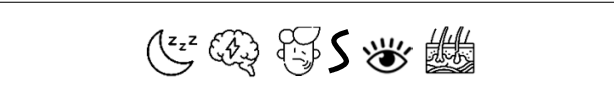 \\
\hline Noonan syndrome [155-157] & Nived 唃 & 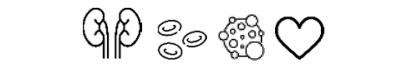 & ID \\
\hline
\end{tabular}


Table 2. Cont.

\begin{tabular}{|c|c|c|c|}
\hline & Endocrine Manifestations & Internal Medicine-Other & Other Disciplines \\
\hline $\begin{array}{l}\text { PNPLA6 gene } \\
\text { mutation [158] }\end{array}$ & 迎角行 & & TID \\
\hline $\begin{array}{l}\text { PTEN hamartoma } \\
\text { tumor syndrome } \\
\text { [159-161] }\end{array}$ & ํㅗㄴ & 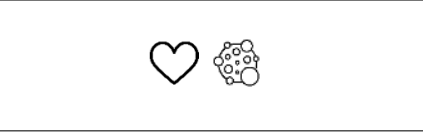 & 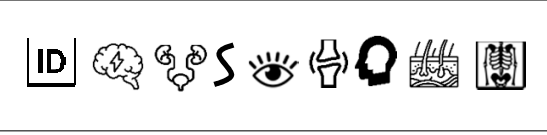 \\
\hline $\begin{array}{l}\text { Prader-Willi (like) } \\
\text { Syndrome } \\
\text { [19,162-171] }\end{array}$ & 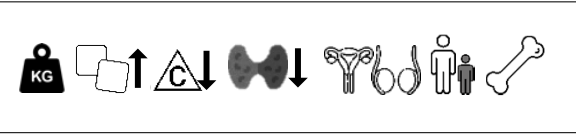 & 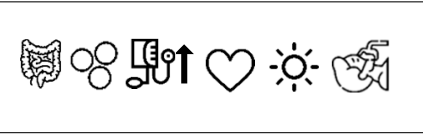 & 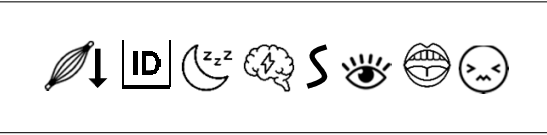 \\
\hline $\begin{array}{l}\text { Rett syndrome } \\
\text { [172-176] }\end{array}$ & 8 & 阔 & 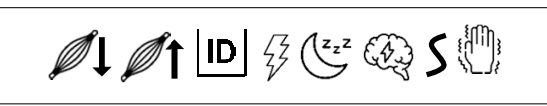 \\
\hline Ring chromosome $21[177,178]$ & 迎魚 & 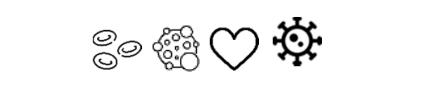 & 个四络 \\
\hline $\begin{array}{l}\text { Saethre-Chotzen } \\
\text { syndrome [179-181] }\end{array}$ & 迎魚 & 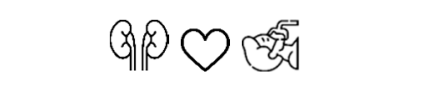 & 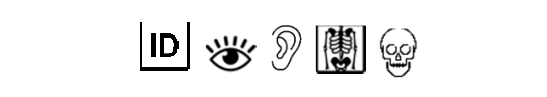 \\
\hline $\begin{array}{l}\text { Say-Barber-Biesecker-Young-Simpson } \\
\text { syndrome (KAT6B mutation) [182-184] }\end{array}$ & $M 1$ & 0 & 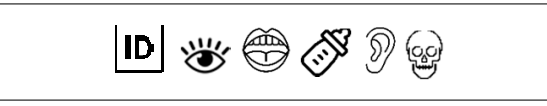 \\
\hline $\begin{array}{l}\text { Sifrim-Hitz-Weiss } \\
\text { Syndrome }[185,186]\end{array}$ & 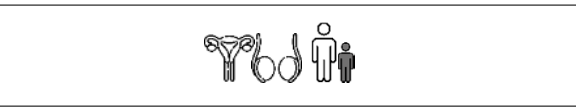 & G/PO & 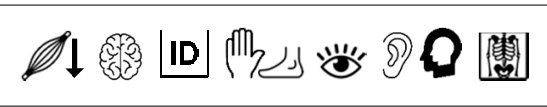 \\
\hline $\begin{array}{c}\text { Silver-Russell } \\
\text { syndrome [82,187-191] }\end{array}$ & 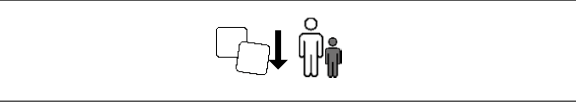 & 阔 & 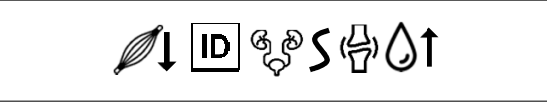 \\
\hline Smith-Lemli-Opitz syndrome [192-196] & $仓 \perp \mathbb{A} \uparrow \lesssim$ & $\circ 8 \sqrt{\kappa^{c o s}} \bigcirc$ & 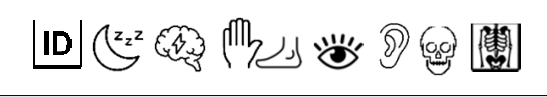 \\
\hline $\begin{array}{c}\text { Smith-Magenis } \\
\text { Syndrome }[82,197]\end{array}$ & 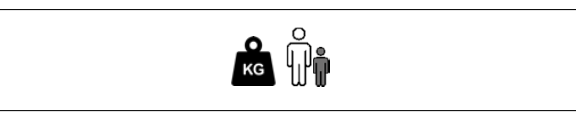 & G/p $\mathrm{O}$ & 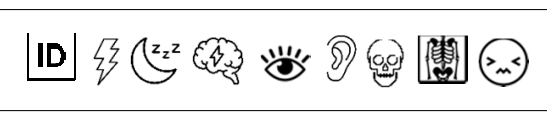 \\
\hline Sotos-like syndrome [198-201] & 醌 & 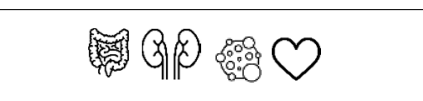 & 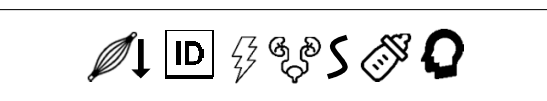 \\
\hline $\begin{array}{c}\text { Tatton-Brown- } \\
\text { Rahman syndrome [202,203] } \\
\end{array}$ & kำ 触 & 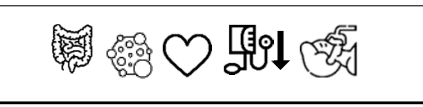 & 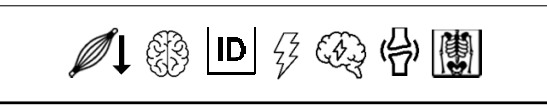 \\
\hline
\end{tabular}


Table 2. Cont.

\begin{tabular}{|c|c|c|c|}
\hline & Endocrine Manifestations & Internal Medicine-Other & Other Disciplines \\
\hline $\begin{array}{l}\text { TBL1X mutation } \\
\text { [204-206] }\end{array}$ & kg $N 1$ & 阔 & 霝 \\
\hline Tetra-X $X$ syndrome $(48, X X X X)$ [207-212] & grabd Sis lis & $\odot \odot$ & 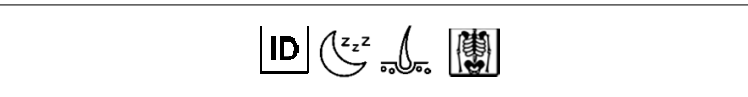 \\
\hline Triple-X syndrome $(47, X X X)[213,214]$ & 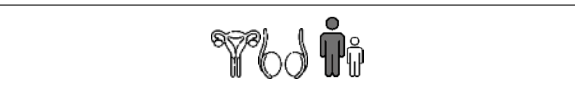 & $Q p$ & 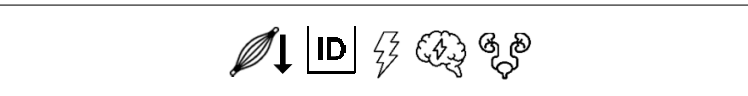 \\
\hline TRPV4 mutation [215] & 蹒 & dêt大 & 迫丝橉 \\
\hline Tuberous sclerosis complex $[216,217]$ & & 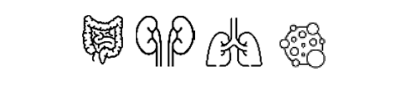 & 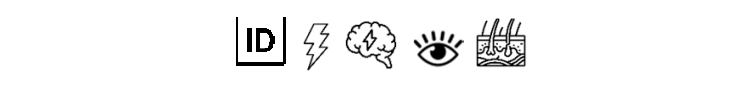 \\
\hline $\begin{array}{l}\text { Turner syndrome } \\
{[218,219]}\end{array}$ & ผ & 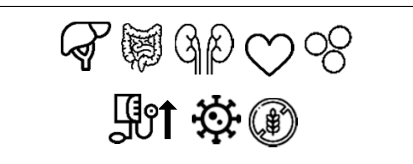 & 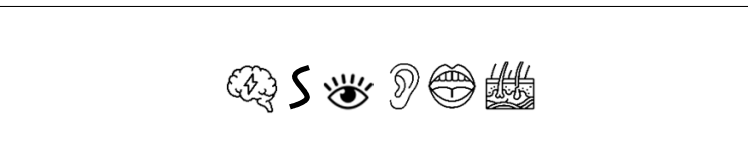 \\
\hline $\begin{array}{l}\text { Williams-Beuren } \\
\text { syndrome [220-223] }\end{array}$ & 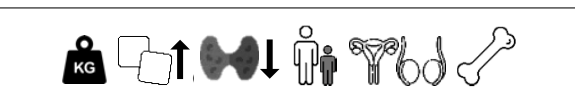 & 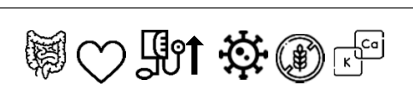 & 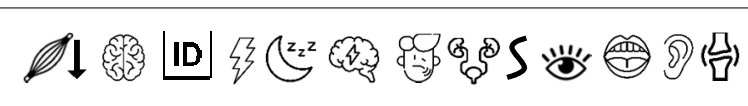 \\
\hline $\begin{array}{c}45, \mathrm{X} / 46, \mathrm{XY} \text { mixed } \\
\text { gonadal dysgenesis }[224,225]\end{array}$ & 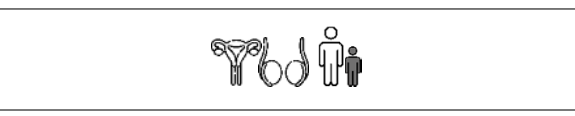 & $Q / p)$ & ID \\
\hline $48, \mathrm{XXXY}$ syndrome [226] & 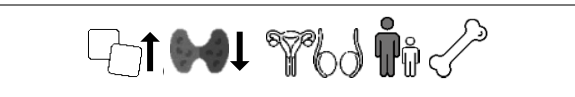 & 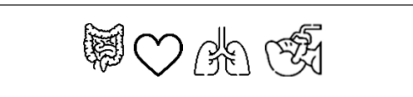 & 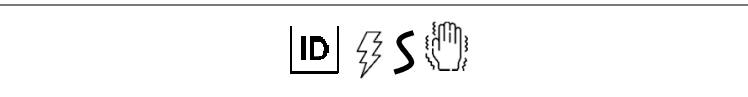 \\
\hline 48,XXYY syndrome [226-229] & 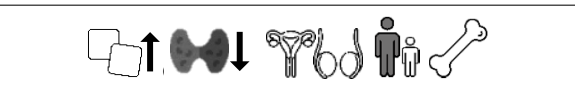 & 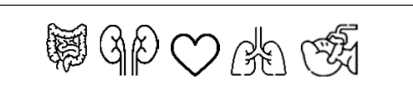 & 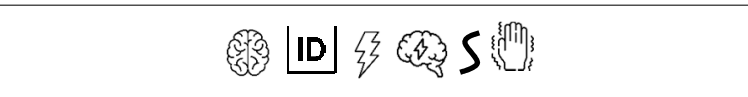 \\
\hline
\end{tabular}




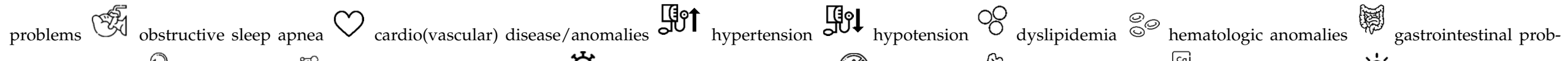

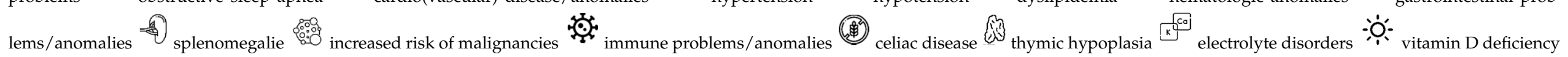

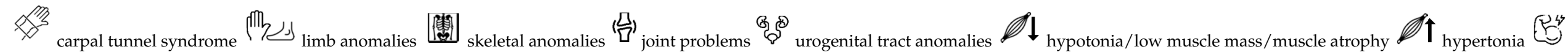

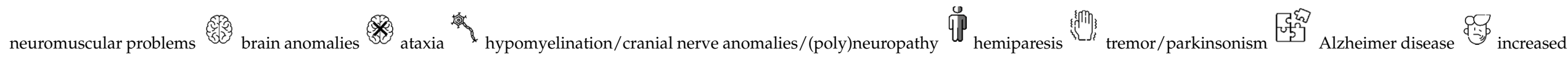

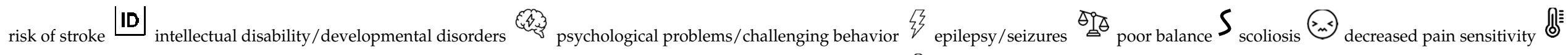

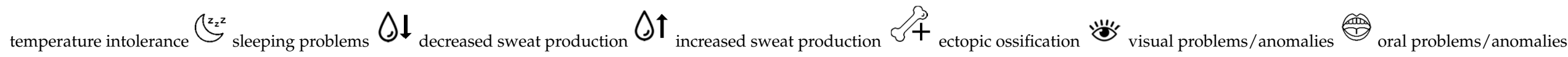

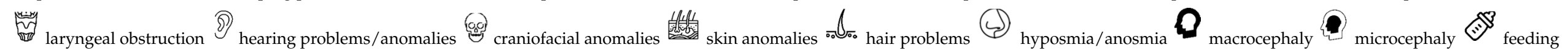

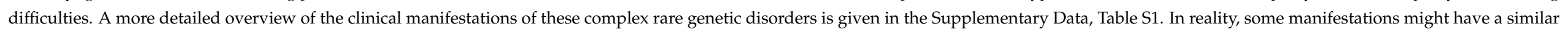

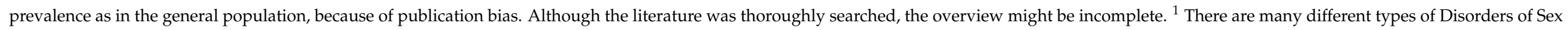

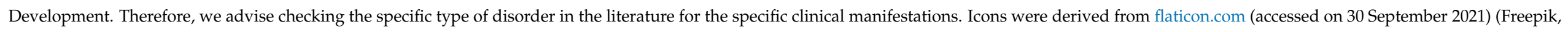

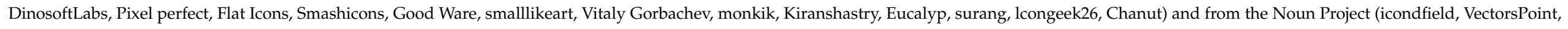
tezar tantular) on 30 September 2021. 
We developed an algorithm for the approach to medical problems in adults with CRGS (Figure 5) and provide an overview of the MD approach to the most common medical problems (fatigue, abdominal complaints, and hypertension) among adults with CRGS (Figures 6-8).

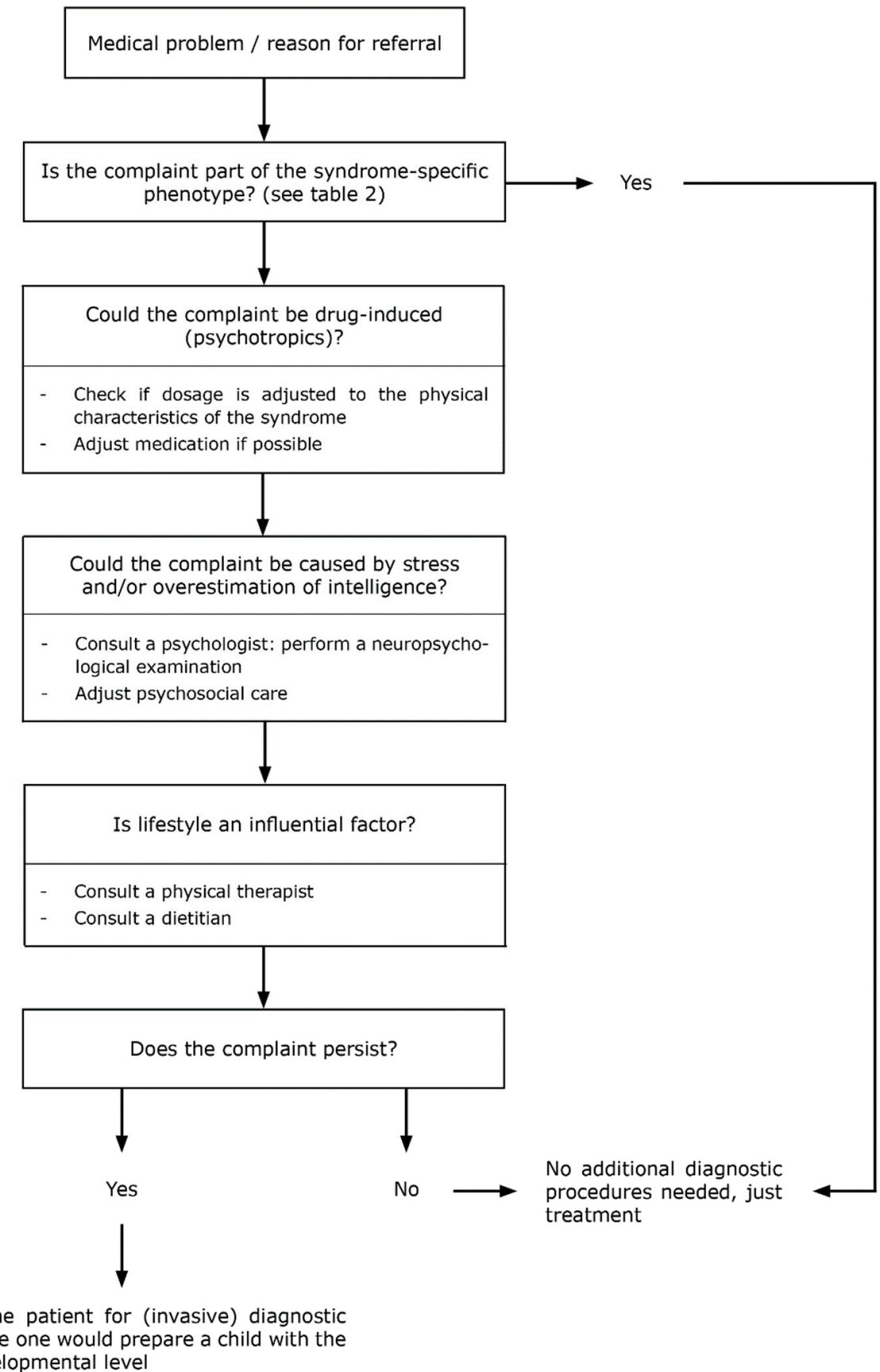

Prepare the patient for (invasive) diagnostic testing, like one would prepare a child with the same developmental level

Figure 5. Algorithm for the approach to medical problems in patients with CRGS. 


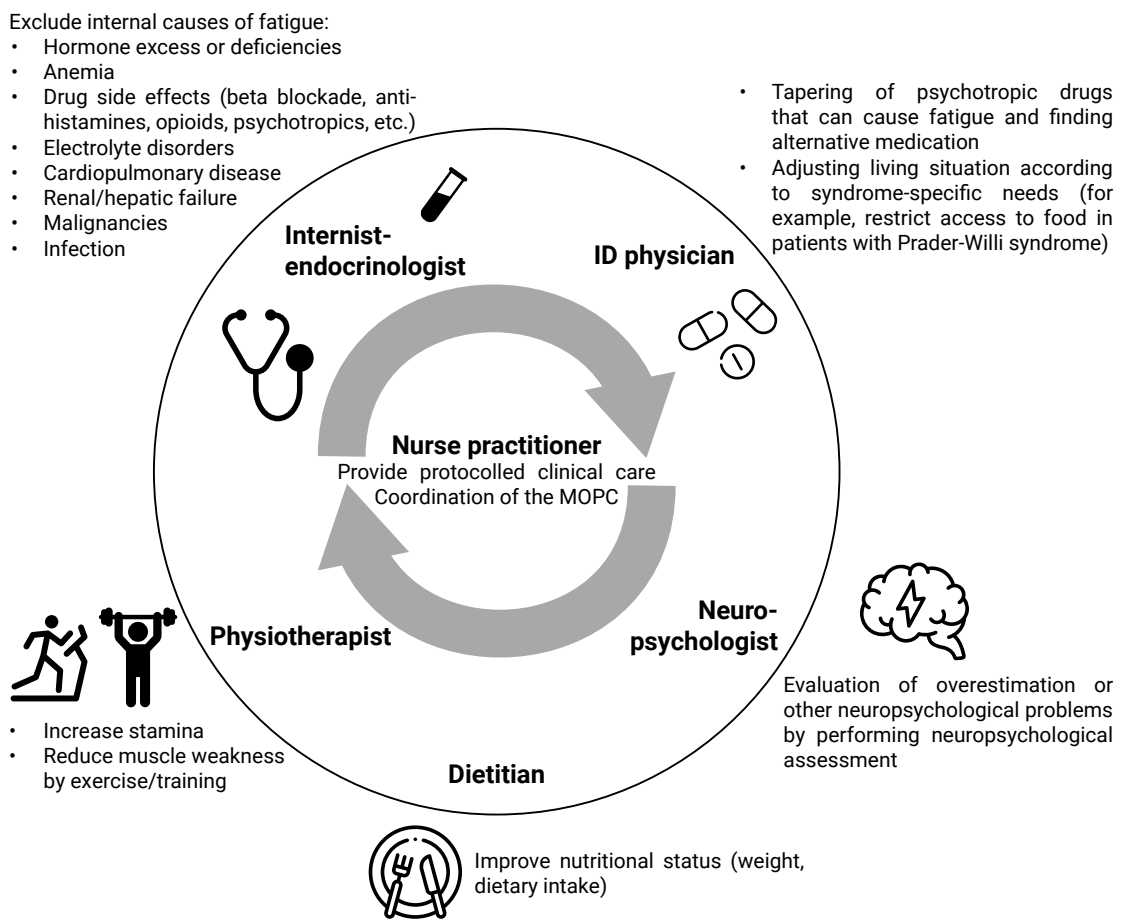

Figure 6. Multidisciplinary approach in our clinic to the patient with a complex rare genetic syndrome and fatigue. Abbreviations: ID, intellectual disability; MOPC, multidisciplinary outpatients' clinic. The figure was designed with resources from flaticon.com (accessed on 30 September 2021) (Freepik, Smashicons).

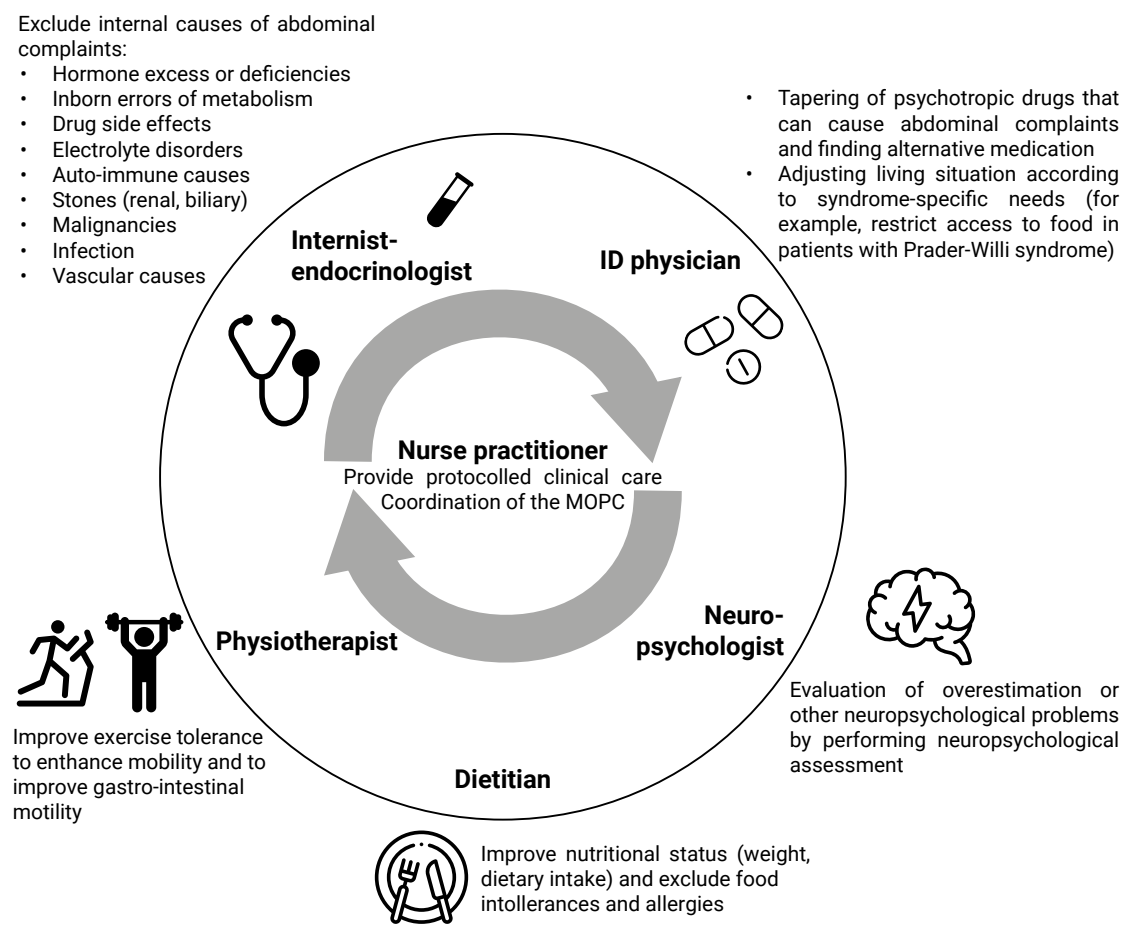

Figure 7. Multidisciplinary approach in our clinic to the patient with a complex rare genetic syndrome and abdominal complaints. Abbreviations: ID, intellectual disability; MOPC, multidisciplinary outpatients' clinic. The figure was designed with resources from flaticon.com (accessed on 30 September 2021) (Freepik, Smashicons). 


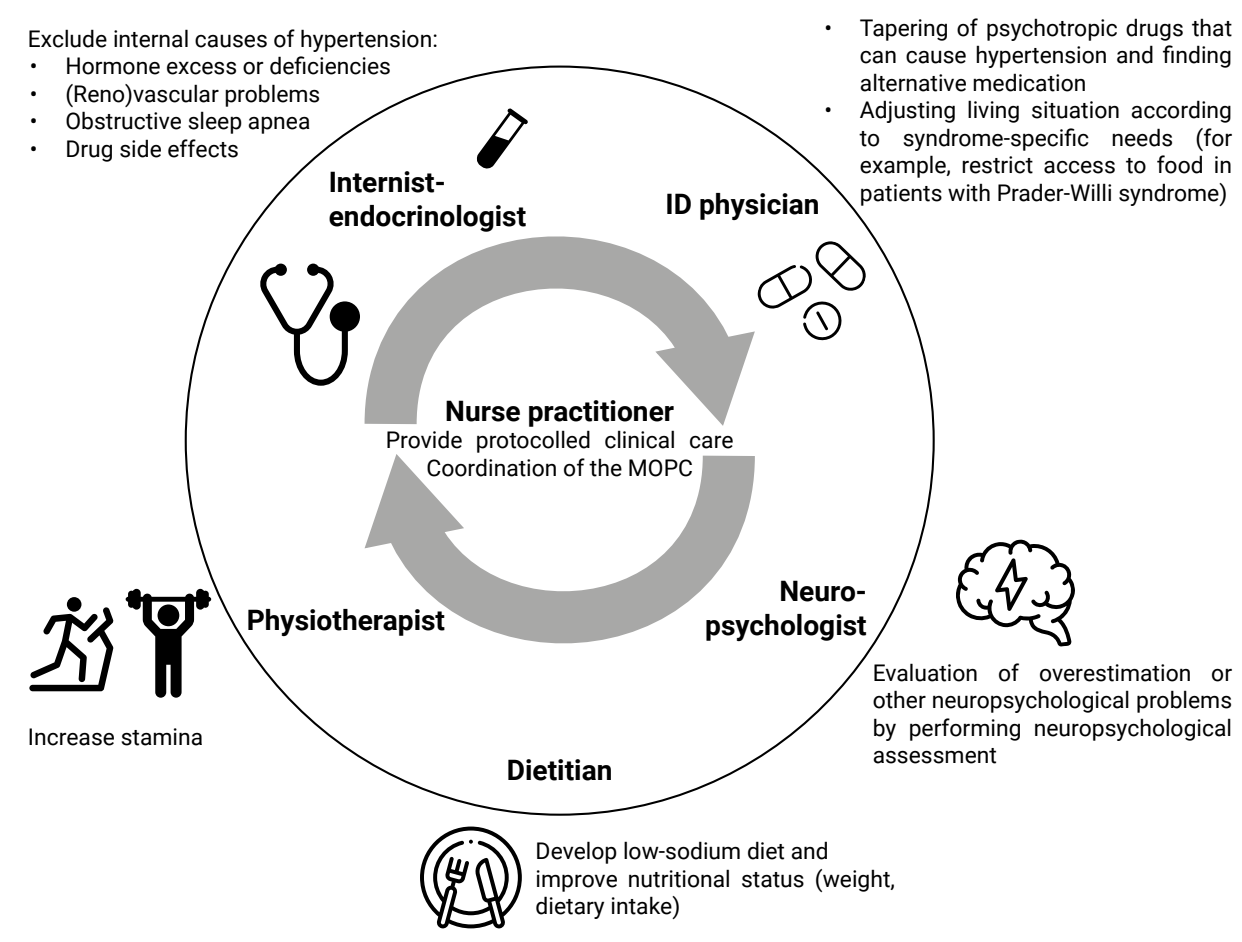

Figure 8. Multidisciplinary approach in our clinic to the patient with a complex rare genetic syndrome and hypertension. Abbreviations: ID, intellectual disability; MOPC, multidisciplinary outpatients' clinic. The figure was designed with resources from flaticon.com (accessed on 30 September 2021) (Freepik, Smashicons).

Among the 720 patients who visited our center, we found several patients with missed diagnoses and patients who had undergone unnecessary diagnostic procedures for symptoms that actually could be explained by their syndrome. The most illustrative cases are described below.

\subsection{Case Series: Missed Diagnoses}

\subsubsection{Case 1: Untreated Diabetes Mellitus in a Patient with PWS}

A 28-year-old male with PWS was referred to our center because of fatigue, weight gain (BMI: $35 \mathrm{~kg} / \mathrm{m}^{2}$ ), and polydipsia. He was seen by a pediatrician until he was 18 years of age. Growth hormone treatment was initiated during puberty and was stopped when the patient reached adult height. He visited the general practitioner (GP) because of fatigue and polydipsia. The GP assumed that polydipsia, like hyperphagia, was part of the PWSspectrum and assumed that fatigue was inherent to PWS. Therefore, he did not perform any further diagnostic testing. The patients' mother was worried about her son and asked for a referral to our center. Our systematic screening revealed that his polydipsia was caused by untreated diabetes mellitus. Furthermore, we found untreated hypogonadism and vitamin D deficiency, which possibly also contributed to his fatigue. We provided the patient and caregivers with diet and exercise recommendations, followed by metformin and liraglutide (sulfonylurea derivatives were not administered due to the possible side effect of weight gain). After normalization of blood glucose levels, polydipsia was no longer a complaint. At follow-up visits, fatigue had also disappeared.

3.1.2. Case 2: Untreated Hypothyroidism, Hypogonadism and Vitamin D Deficiency in a Patient with PWS

A 23-year-old male with PWS visited the GP because of weight gain (BMI: $32.5 \mathrm{~kg} / \mathrm{m}^{2}$ ), fatigue, passive behavior, and poor exercise tolerance. The GP performed a physical examination and biochemical analysis, including TSH to screen for hypothyroidism. TSH was normal; other hormone or vitamin deficiencies were not included in the GP's screen- 
ing. Unaware of the fact that hypothyroidism in PWS often has a central origin, the GP concluded that hypothyroidism was absent and that symptoms of fatigue and passive behavior were part of the PWS-phenotype. Due to persistent complaints, the patient visited our MOPC. As a normal TSH does not rule out central hypothyroidism associated with PWS, we measured free T4 and found severe untreated hypothyroidism. Our screening also revealed undiagnosed hypogonadism and vitamin D deficiency. After replacement with thyroid hormone, testosterone, and vitamin $\mathrm{D}$, exercise tolerance improved and the patient lost $30 \mathrm{~kg}$ of weight (BMI: $23.4 \mathrm{~kg} / \mathrm{m}^{2}$ ). Fatigue was no longer present and his passive behavior disappeared.

\subsubsection{Case 3: Untreated Heart Failure in a Patient with PWS}

A 28-year-old woman with PWS suffered from weight gain and leg edema. NTproBNP (N-terminal pro-brain natriuretic peptide) levels measured by the internist were normal and cardiac ultrasound detected no abnormalities. Unaware of the fact that cardiac ultrasound and NT-proBNP are unreliable in PWS (NT-proBNP can be false negative in up to $15 \%$ of obese patients [230]), the internist concluded that the patient's symptoms were not caused by any cardiac problem. Shortly after visiting the internist, the patient developed severe shortness of breath and was admitted to the intensive care unit with respiratory insufficiency due to cardiac failure. After adequate treatment of the heart failure, the patient is doing well.

\subsection{Case Series: Overtreatment}

\subsubsection{Case 4: Hypersexuality in a Patient with Cockayne Syndrome}

A 20-year-old male with Cockayne syndrome was referred to our MOPC because of hypersexual behavior. The patient was on the waiting list to undergo a bilateral orchidectomy because of his hypersexuality with inappropriate sexual behavior. His medication list revealed that he was using methylphenidate. The treating physician at that time assumed that hypersexual behavior was part of the Cockayne phenotype. Hypersexuality is, however, not part of the spectrum of Cockayne syndrome. We, therefore, searched for alternative explanations, such as drug side effects. As increased libido can be a side effect of methylphenidate, this was tapered. His hypersexuality disappeared and the bilateral orchidectomy was canceled.

\subsubsection{Case 5: Overtreatment with Hydrocortisone in a Patient with PWS}

A 30-year-old male with PWS was referred to our MOPC due to progressive obesity. A few years earlier, he had been diagnosed with central adrenal insufficiency (CAI), based on low morning cortisol found by an internist in a regional hospital. The patient had been taking daily hydrocortisone ever since and had gained $2 \mathrm{~kg}$ per year. He presented at our clinic with a BMI of $32 \mathrm{~kg} / \mathrm{m}^{2}$. As CAI is extremely rare in adults with PWS (even in patients with low morning cortisol [164]), we gradually tapered the hydrocortisone. One year after stopping hydrocortisone, BMI had returned to normal. A metyrapone test confirmed the absence of CAI.

\subsection{Case Series: Unnecessary Diagnostic Tests}

3.3.1. Case 6: Depression and Behavioral Problems in a Patient with PWS

A 47-year-old male with PWS was referred to our clinic due to frequent falls and apathy, with the request to rule out underlying somatic pathology. The patient had visited a neurologist to rule out neurologic pathology. A brain CT had not revealed any abnormalities. He had also visited a cardiologist who had performed an electrocardiogram, cardiac ultrasound, and blood tests, which were all normal.

In the past, the GP had prescribed high dosages of pipamperon for temper outbursts, after which the patient had developed epileptic seizures. Subsequently, a neurologist had prescribed levetiracetam to treat the epileptic seizures. As notable side effects of pipamperon and levetiracetam include sleepiness and depression [231], we suspected 
that his apathy could be caused by the high dosage of psychotropic drugs. As the initial temper outbursts appeared to be triggered by stress, we advised adapting the (intensive) daycare program, after which temper outbursts were no longer a problem. Pipamperon and levetiracetam were gradually tapered, after which his apathy disappeared.

3.3.2. Case 7: Unnecessary Invasive Testing (Colonoscopy and Magnetic Resonance Cholangio-Pancreatography (MRCP)) in a Patient with Williams-Beuren Syndrome

A 52-year-old woman with Williams-Beuren Syndrome (WBS) was referred to our MOPC by the clinical geneticist. She had an intellectual disability with a developmental age of an 8-year-old child. Her medical history revealed hypertension for which she was taking three different antihypertensive drugs. When asking for stress as a potential cause of her hypertension, the patient became emotional and reported having undergone several invasive diagnostic tests which she had experienced as very traumatic. She had undergone a colonoscopy to find the cause of her diarrhea. She had also undergone an MRCP to find the cause for mild liver dysfunction. As our screening revealed undiagnosed and untreated diabetes mellitus type II, we concluded that her mild liver dysfunction was probably caused by diabetes mellitus related non-alcoholic fatty liver disease. Diarrhea turned out to be a side effect of the medication she was using (pancreatin). Colonoscopy and MRCP had been carried out according to regular procedures for adults with normal intelligence. The patient had not been able to process the information provided before and during these invasive procedures. As patients with WBS have remarkably strong verbal skills, over-estimation of cognitive capacities is a common pitfall in patients with WBS [232]. The physician, unaware of the impact that these invasive tests could have on the mental health of the patient, had not linked the hypertension to previous invasive procedures. We referred the patient to the psychologist for psychological assistance and trauma treatment, after which the anti-hypertensive drugs could be successfully tapered.

\section{Discussion}

We report our five-year experience of 'internal medicine-endocrinology for adults with complex rare genetic syndromes'. Illustrated by a population overview and case series, we show that missed diagnoses, undertreatment, overtreatment, and unnecessary diagnostic tests seem common among adults with CRGS. We hypothesize that this is largely due to a lack of syndrome-specific knowledge among internists-endocrinologists and GPs.

The lack of syndrome knowledge is not surprising, as internists-endocrinologists hardly encountered any adults with rare genetic syndromes until recently. However, due to increased life expectancy, a growing number of patients with CRGS is now reaching adult age. Therefore, it is important to raise awareness of syndrome-specific manifestations, medical needs, and diagnostic pitfalls of this vulnerable population. The most frequent physical complaints in our population were fatigue $(60 \%)$ and abdominal complaints (37\%). Furthermore, many patients had hypertension (20\%). These medical problems were much more frequent in adults with CRGS than in the general population, where fatigue is present in 31\% [233], abdominal complaints in 2-4\% [234], and hypertension in 5-10\% [235]. However, it must be noted that patients are only referred to our center in case of (suspected) pathology in the internal medicine domain. This might result in a higher prevalence of hypertension, fatigue, and abdominal complaints.

For this paper, we mainly focused on the recognition of and approach to physical complaints in adults with CRGS. However, early diagnosis of complications associated with the different syndromes, as displayed in Table 2, is also an important aspect of clinical care for adults with CRGS. In our center, we screen for these complications with a systematic health screening [19]. Further research should also focus on how to improve the early diagnosis of complications associated with CRGS. Furthermore, for the case series, the number of cases about PWS is higher than one would expect, based on the incidence of PWS (1:16,000-21,000 live births [236,237]). Since our center is the only Reference Center for adults with PWS in the Netherlands, a relatively high number of adults with PWS are treated at our center, which resulted in more cases of Prader-Willi syndrome. 
Based on our experience with more than seven hundred CRGS adults, we provide two main clinical recommendations which will help other internist-endocrinologists to provide good clinical care to this vulnerable patient population.

\subsection{Multidisciplinary Approach}

Our first recommendation is to treat adults with CRGS in a multidisciplinary setting. As CRGS patients often have multiple health problems simultaneously, a multidisciplinary team should ideally consist of an internist-endocrinologist, psychologist, nurse practitioner, dietitian, physiotherapist, clinical geneticist, and ID physician. If it is not possible to include an ID physician in the team, a behavioral expert or psychiatrist should be included to address behavioral issues. Ideally, a nurse practitioner should be part of the multidisciplinary team, to coordinate the MD care and provide protocolled clinical care for (relatively) high-prevalence syndromes.

Due to the multi-organ involvement of many of the syndromes, it is important to have direct lines of communication with other specialists, such as cardiologists, gynecologists, urologists, neurologists, and dermatologists, and include them in the MD team when needed.

The MD approach of the most common complaints in our cohort (fatigue and abdominal complaints) and hypertension is shown in Figures 6-8.

Although organizing MD care might seem expensive, it will probably be cost-efficient in the long term. The social-economic burden of rare diseases in Europe, including PWS, is high $[238,239]$. One way of reducing the high annual economic burden (estimated to be around EUR 60.000 per patient) for patients with PWS [240] is improving clinical care by establishing MOPCs. Several studies have shown that MOPCs are cost-effective for diseases such as heart failure, kidney disease, and diabetes mellitus [241,242]. Although to our knowledge no studies were performed to date on the cost-effectiveness of MOPCs for CRGS, they will likely reduce costs in a similar manner by preventing overtreatment, unnecessary diagnostic tests, and medical complications. Although the annual economic burden will likely decrease, the total economic burden might be similar, due to the increased number of life-years as a result of the improved MD care. However, this will probably be compensated by the expected improved quality of life in adults with CRGS.

A number of adult dedicated clinics have been established in order to treat adults with specific 'higher prevalence' syndromes, such as Down syndrome [243,244] and Turner syndrome [245-248]. However, MD care for patients with (ultra-)rare syndromes was not available yet. Therefore, in our center, we have organized MD care for all adults with CRGS, including extremely rare syndromes. To our knowledge, our multidisciplinary center for CRGS is the first worldwide, in the field of internal medicine-endocrinology. It must be noted, however, that besides establishing an MD center for CRGS, the transition from pediatric to adult care should be optimized to prevent fragmented and inadequate care.

The multidisciplinary aspect of the healthcare we provide, not only has medical advantages; it is also much appreciated by the patients and their caregivers as shown by a satisfaction survey that was performed during the first year of the MOPC. Meeting all necessary disciplines during one visit saves them time, effort, stress, and money that would otherwise be spent on traveling to separate hospital visits.

\subsection{Clinical Algorithm for the Approach to Physical Symptoms}

Our second recommendation is to use a clinical algorithm we have developed for the approach to physical symptoms in adults with CRGS (Figure 5). This approach differs from regular internal medicine patient care due to syndrome-specific health problems, but also due to the increased prevalence of polypharmacy and stress and unfavorable lifestyle related to intellectual disability and/or challenging behavior often present in patients with CRGS. 


\subsubsection{Syndrome-Specific Complaints}

For any complaint the patient presents with, the internist-endocrinologist or nurse practitioner should always first check whether the problem could be inherent to the syndrome (Table 2).

\subsubsection{Drug Side Effects}

If a complaint or symptom is not part of the syndrome, one should check whether it could be a drug-related side effect. As polypharmacy is common in adults with CRGS, drug-related side effects should be considered as the cause of any potential complaint. Especially psychotropic drugs are known to cause fatigue, abdominal complaints, and hypertension [249]. When the presenting complaint or symptom is indeed caused by psychotropic drugs, tapering the dose, or finding alternative medication should always be done in consultation with an ID physician or psychiatrist.

\subsubsection{Chronic Stress}

Once drug-related side effects have been excluded, one should consider whether the presenting complaint or symptom might be caused by chronic stress. In our experience, overestimation of cognitive capacities is a frequent cause of chronic stress and stress-related physical complaints in patients with (unrecognized) intellectual disabilities. Especially in syndromes where verbal intelligence exceeds performance intelligence (for example in PWS or WBS), patients are easily overestimated. If overestimation is indeed suspected, neuropsychologists can perform neuropsychologic assessments to assess the actual cognitive capacities. The ID-physician can help residential homes in adjusting the support and living situation of the patient to their IQ and syndrome-specific needs. For example, restriction of access to food can prevent a lot of stress in patients with PWS who are (due to their hyperphagia) continuously foraging and bargaining for food. The behavioral therapist can subsequently provide training to the caregivers to improve patient-caregiver interaction and address any potential stress-related issues. Moreover, if the patient has no intellectual disability, chronic stress can occur due to syndrome-related impaired executive functions or other psychological and contextual factors. In that case, the support of a neuropsychologist or behavioral therapist is also warranted.

\subsubsection{Lifestyle}

If the complaint can be improved by lifestyle or other contextual modifications, a dietitian or physiotherapist (specialized in patients with intellectual disability when necessary) should be consulted. Especially in large residential homes, physical activity and nutrition are often inadequate [250]. In the case of physical or intellectual disabilities, lifestyle interventions should be customized to the capacities of the individual patient [251].

\subsubsection{Additional Diagnostic Testing}

Only if the symptom is not part of the syndrome, nor related to polypharmacy, intellectual disability, behavioral factors, and/or lifestyle, or persists despite adequate treatment, underlying organic causes should be excluded by diagnostic tests. If diagnostic procedures are needed, adults with intellectual disabilities should be informed in a way that is appropriate for their developmental age, to prevent traumatic experiences.

\section{Conclusions}

Our experience with 720 adults with CRGS illustrates that the medical management of CRGS adults poses significant challenges for healthcare professionals. The complex medical and neuropsychiatric comorbidity can lead to missed diagnoses on the one hand, and to unnecessary invasive procedures on the other hand. As more and more CRGS patients are now reaching adult age and making the transfer to internal medicine/adult endocrinology, internist-endocrinologists should be aware of the medical pitfalls and the special needs of adults with CRGS. 
Based on our findings and the literature, we recommend treating adults with CRGS in a multidisciplinary team and using the clinical algorithm we have developed for the approach to physical symptoms in adults with CRGS.

As knowledge about syndrome-specific health problems is crucial to prevent the personal and financial burden of unnecessary diagnostics and under- and overtreatment, we believe that education about syndrome-specific health problems in adults with CRGS should be part of the internist-endocrinologists' medical training.

Supplementary Materials: The following are available online at https: / www.mdpi.com/article/10.3 390/jcm10225457/s1, Table S1: Clinical manifestations of complex rare genetic disorders seen in our center since 2015 .

Author Contributions: Conceptualization, A.G.W.R. and L.C.G.d.G.; methodology, A.G.W.R. and L.C.G.d.G.; formal analysis, A.G.W.R.; investigation, A.G.W.R.; resources, L.C.G.d.G.; data curation, A.G.W.R., M.R.A.P. and L.C.G.d.G.; writing-original draft preparation, A.G.W.R. and M.R.A.P.; writing-review and editing, A.G.W.R., M.R.A.P., K.P., K.D., A.A.K.-M., R.K., A.G.B.-R., A.v.E., J.M.C.V., J.J.v.d.M., N.v.A.-v.W., F.M.E.H., A.J.v.d.L. and L.C.G.d.G.; visualization, A.G.W.R.; supervision, L.C.G.d.G. and A.J.v.d.L.; project administration, L.C.G.d.G.; funding acquisition, L.C.G.d.G. All authors have read and agreed to the published version of the manuscript.

Funding: This research received no external funding.

Institutional Review Board Statement: The study was conducted according to the guidelines of the Declaration of Helsinki. Ethical review and approval were waived for this study by the Institutional Review Board (or Ethics Committee) of the Erasmus University Medical Center (protocol code MEC-2018-1389, 24 September 2018), as this is not applicable for retrospective data collection from patient records.

Informed Consent Statement: Informed consent was obtained from subjects involved in the study or anonymized patient data was collected.

Data Availability Statement: Some or all datasets generated during and/or analyzed during the current study are not publicly available but are available from the corresponding author on reasonable request.

Acknowledgments: The authors wish to thank E. Krabbendam from the Erasmus MC Medical Library for developing the search strategy.

Conflicts of Interest: The authors declare no conflict of interest.

\section{References}

1. Valk, H.M.J.V.S.L.-D.; Walsh, P.N. Managing health problems in people with intellectual disabilities. BMJ 2008, 337 , a2507. [CrossRef]

2. $\quad$ Liptak, G.S.; O’Donnell, M.; Conaway, M.; Chumlea, W.C.; Wolrey, G.; Henderson, R.C.; Fung, E.; Stallings, V.A.; Samson-Fang, L.; Calvert, R.; et al. Health status of children with moderate to severe cerebral palsy. Dev. Med. Child Neurol. 2001, 43, 364-370. [CrossRef] [PubMed]

3. Oeseburg, B.; Dijkstra, G.J.; Groothoff, J.W.; Reijneveld, S.A.; Jansen, D.E. Prevalence of chronic health conditions in children with intellectual disability: A systematic literature review. Intellect. Dev. Disabil. 2011, 49, 59-85. [CrossRef] [PubMed]

4. Sarneel, M.C.; Penning, C.; Roukema, J.; Moll, H.A.; Bindels-de Heus, G.C.B.; Evenhuis, H.M. Inventory of frequency and reasons of visits of children with profound intellectual and multiple disabilities to a university hospital. Ned. Tijdschr. Voor Kindergeneeskd. $2005,73,32-33$.

5. Strauss, D.; Brooks, J.; Rosenbloom, L.; Shavelle, R. Life expectancy in cerebral palsy: An update. Dev. Med. Child Neurol. 2008, 50, 487-493. [CrossRef] [PubMed]

6. Westbom, L.; Bergstrand, L.; Wagner, P.; Nordmark, E. Survival at 19 years of age in a total population of children and young people with cerebral palsy. Dev. Med. Child Neurol. 2011, 53, 808-814. [CrossRef]

7. Bhaumik, S.; Watson, J.; Barrett, M.; Raju, B.; Burton, T.; Forte, J. Transition for teenagers with intellectual disability: Carer's perspectives. J. Policy Pract. Intellect. Disabil. 2011, 8, 53-61. [CrossRef]

8. Bindels-de Heus, K.G.; van Staa, A.; van Vliet, I.; Ewals, F.V.; Hilberink, S.R. Transferring young people with profound intellectual and multiple disabilities from pediatric to adult medical care: Parents' experiences and recommendations. Intellect. Dev. Disabil. 2013, 51, 176-189. [CrossRef]

9. Bruin, G. The Transition of Young Persons with Profound Multiple Disabilities from Pediatric to Adult Care; Rotterdam University: Rotterdam, The Netherlands, 2008. 
10. Inspectie voor de Volksgezondheid (IGZ). Profound Intellectual and Multiple Disabilities: What Next? A Research into the Quality of Care for People with PIMD; IGZ (Health Care Inspectorate): The Hague, The Netherlands, 2000.

11. Neece, C.L.; Kraemer, B.R.; Blacher, J. Transition satisfaction and family well being among parents of young adults with severe intellectual disability. Intellect. Dev. Disabil. 2009, 47, 31-43. [CrossRef]

12. Schrander-Stumpel, C.T.; Sinnema, M.; van den Hout, L.; Maaskant, M.A.; van Schrojenstein Lantman-de Valk, H.M.; Wagemans, A.; Schrander, J.J.; Curfs, L.M. Healthcare transition in persons with intellectual disabilities: General issues, the Maastricht model, and Prader-Willi syndrome. Am. J. Med. Genet. C Semin. Med. Genet. 2007, 145, 241-247. [CrossRef]

13. Stewart, D. Transition to adult services for young people with disabilities: Current evidence to guide future research. Dev. Med. Child Neurol. 2009, 51 (Suppl. 4), 169-173. [CrossRef]

14. van Staa, A.L.; Jedeloo, S.; van Meeteren, J.; Latour, J.M. Crossing the transition chasm: Experiences and recommendations for improving transitional care of young adults, parents and providers. Child Care Health Dev. 2011, 37, 821-832. [CrossRef]

15. Young, N.L.; Barden, W.S.; Mills, W.A.; Burke, T.A.; Law, M.; Boydell, K. Transition to adult-oriented health care: Perspectives of youth and adults with complex physical disabilities. Phys. Occup. Ther. Pediatr. 2009, 29, 345-361. [CrossRef] [PubMed]

16. Both, P.; Ten Holt, L.; Mous, S.; Patist, J.; Rietman, A.; Dieleman, G.; Ten Hoopen, L.; Vergeer, M.; de Wit, M.C.; Bindels-de Heus, K.; et al. Tuberous sclerosis complex: Concerns and needs of patients and parents from the transitional period to adulthood. Epilepsy Behav. 2018, 83, 13-21. [CrossRef] [PubMed]

17. Rietman, A.B.; van Helden, H.; Both, P.H.; Taal, W.; Legerstee, J.S.; van Staa, A.; Moll, H.A.; Oostenbrink, R.; van Eeghen, A.M. Worries and needs of adults and parents of adults with neurofibromatosis type 1. Am. J. Med. Genet. Part A 2018, 176, 1150-1160. [CrossRef] [PubMed]

18. European Commission. Rare Diseases. 2021. Available online: https://ec.europa.eu/health/non_communicable_diseases/rare_ diseases_en (accessed on 12 November 2021).

19. Pellikaan, K.; Rosenberg, A.G.W.; Kattentidt-Mouravieva, A.A.; Kersseboom, R.; Bos-Roubos, A.G.; Veen-Roelofs, J.M.C.; van Wieringen, N.; Hoekstra, F.M.E.; van den Berg, S.A.A.; van der Lely, A.J.; et al. Missed Diagnoses and Health Problems in Adults With Prader-Willi Syndrome: Recommendations for Screening and Treatment. J. Clin. Endocrinol. Metab. 2020, 105, e4671-e4687. [CrossRef] [PubMed]

20. Mantovani, G.; Bastepe, M.; Monk, D.; de Sanctis, L.; Thiele, S.; Usardi, A.; Ahmed, S.F.; Bufo, R.; Choplin, T.; De Filippo, G.; et al. Diagnosis and management of pseudohypoparathyroidism and related disorders: First international Consensus Statement. Nat. Rev. Endocrinol. 2018, 14, 476-500. [CrossRef]

21. Joseph, A.W.; Shoemaker, A.H.; Germain-Lee, E.L. Increased prevalence of carpal tunnel syndrome in albright hereditary osteodystrophy. J. Clin. Endocrinol. Metab. 2011, 96, 2065-2073. [CrossRef]

22. Schwartz, C.E.; Stevenson, R.E. The MCT8 thyroid hormone transporter and Allan-Herndon-Dudley syndrome. Best Pract. Res. Clin. Endocrinol. Metab. 2007, 21, 307-321. [CrossRef]

23. Remerand, G.; Boespflug-Tanguy, O.; Tonduti, D.; Touraine, R.; Rodriguez, D.; Curie, A.; Perreton, N.; Des Portes, V.; Sarret, C.; Group, R.A.S. Expanding the phenotypic spectrum of Allan-Herndon-Dudley syndrome in patients with SLC16A2 mutations. Dev. Med. Child Neurol. 2019, 61, 1439-1447. [CrossRef]

24. Ozantürk, A.; Marshall, J.D.; Collin, G.B.; Düzenli, S.; Marshall, R.P.; Candan, Ş.; Tos, T.; Esen, İ; Taşkesen, M.; Çayır, A.; et al. The phenotypic and molecular genetic spectrum of Alström syndrome in 44 Turkish kindreds and a literature review of Alström syndrome in Turkey. J. Hum. Genet. 2015, 60, 1-9. [CrossRef] [PubMed]

25. Álvarez-Satta, M.; Castro-Sánchez, S.; Valverde, D. Alström syndrome: Current perspectives. Appl. Clin. Genet. 2015, 8, 171-179. [PubMed]

26. Larson, A.M.; Shinnick, J.E.; Shaaya, E.A.; Thiele, E.A.; Thibert, R.L. Angelman syndrome in adulthood. Am. J. Med. Genet. Part A 2015, 167, 331-344. [CrossRef] [PubMed]

27. Smith, J.C. Angelman syndrome: Evolution of the phenotype in adolescents and adults. Dev. Med. Child Neurol. 2001, 43, 476-480. [CrossRef]

28. Seifi, M.; Walter, M.A. Axenfeld-Rieger syndrome. Clin. Genet. 2018, 93, 1123-1130. [CrossRef]

29. Jena, A.K.; Kharbanda, O.P. Axenfeld-Rieger syndrome: Report on dental and craniofacial findings. J. Clin. Pediatr. Dent. 2005, 30, 83-88. [CrossRef]

30. Priya, S.; Nampoothiri, S.; Sen, P.; Sripriya, S. Bardet-Biedl syndrome: Genetics, molecular pathophysiology, and disease management. Indian. J. Ophthalmol. 2016, 64, 620-627. [CrossRef]

31. Mujahid, S.; Hunt, K.F.; Cheah, Y.S.; Forsythe, E.; Hazlehurst, J.M.; Sparks, K.; Mohammed, S.; Tomlinson, J.W.; Amiel, S.A.; Carroll, P.V.; et al. The Endocrine and Metabolic Characteristics of a Large Bardet-Biedl Syndrome Clinic Population. J. Clin. Endocrinol. Metab. 2018, 103, 1834-1841. [CrossRef]

32. Guran, T.; Ekinci, G.; Atay, Z.; Turan, S.; Akcay, T.; Bereket, A. Radiologic and hormonal evaluation of pituitary abnormalities in patients with Bardet-Biedl syndrome. Clin. Dysmorphol. 2011, 20, 26-31. [CrossRef]

33. Shrestha, S.; Chaudhary, N. A rare case of obesity. Can it be Bardet-Biedl Syndrome? Clin. Case Rep. 2019, 7, 1725-1728. [CrossRef]

34. Beales, P.L.; Elcioglu, N.; Woolf, A.S.; Parker, D.; Flinter, F.A. New criteria for improved diagnosis of Bardet-Biedl syndrome: Results of a population survey. J. Med. Genet. 1999, 36, 437-446. [PubMed]

35. Cunniff, C.; Djavid, A.R.; Carrubba, S.; Cohen, B.; Ellis, N.A.; Levy, C.F.; Jeong, S.; Lederman, H.M.; Vogiatzi, M.; Walsh, M.F.; et al. Health supervision for people with Bloom syndrome. Am. J. Med. Genet. Part A 2018, 176, 1872-1881. [CrossRef] 
36. Gécz, J.; Turner, G.; Nelson, J.; Partington, M. The Börjeson-Forssman-Lehman syndrome (BFLS, MIM \#301900). Eur. J. Hum. Genet. 2006, 14, 1233-1237. [CrossRef]

37. Lower, K.M.; Solders, G.; Bondeson, M.-L.; Nelson, J.; Brun, A.; Crawford, J.; Malm, G.; Börjeson, M.; Turner, G.; Partington, M.; et al. $1024 \mathrm{C}>\mathrm{T}(\mathrm{R} 342 \mathrm{X})$ is a recurrent PHF6 mutation also found in the original Börjeson-Forssman-Lehmann syndrome family. Eur. J. Hum. Genet. 2004, 12, 787-789. [CrossRef] [PubMed]

38. Küry, S.; van Woerden, G.M.; Besnard, T.; Proietti Onori, M.; Latypova, X.; Towne, M.C.; Cho, M.T.; Prescott, T.E.; Ploeg, M.A.; Sanders, S.; et al. De Novo Mutations in Protein Kinase Genes CAMK2A and CAMK2B Cause Intellectual Disability. Am. J. Hum. Genet. 2017, 101, 768-788. [CrossRef] [PubMed]

39. Chia, P.H.; Zhong, F.L.; Niwa, S.; Bonnard, C.; Utami, K.H.; Zeng, R.; Lee, H.; Eskin, A.; Nelson, S.F.; Xie, W.H.; et al. A homozygous loss-of-function CAMK2A mutation causes growth delay, frequent seizures and severe intellectual disability. Elife 2018, 7, e32451. [CrossRef] [PubMed]

40. Gregory, L.C.; Gevers, E.F.; Baker, J.; Kasia, T.; Chong, K.; Josifova, D.J.; Caimari, M.; Bilan, F.; McCabe, M.J.; Dattani, M.T. Structural pituitary abnormalities associated with CHARGE syndrome. J. Clin. Endocrinol. Metab. 2013, 98, E737-E743. [CrossRef]

41. Wheeler, P.G.; Quigley, C.A.; Sadeghi-Nejad, A.; Weaver, D.D. Hypogonadism and CHARGE association. Am. J. Med. Genet. 2000, 94, 228-231. [CrossRef]

42. Forward, K.E.; Cummings, E.A.; Blake, K.D. Risk factors for poor bone health in adolescents and adults with CHARGE syndrome. Am. J. Med. Genet. Part A 2007, 143, 839-845. [CrossRef]

43. Macdonald, M.; Hudson, A.; Bladon, A.; Ratcliffe, E.; Blake, K. Experiences in feeding and gastrointestinal dysfunction in children with CHARGE syndrome. Am. J. Med. Genet. Part A 2017, 173, 2947-2953. [CrossRef]

44. Hudson, A.; Macdonald, M.; Friedman, J.N.; Blake, K. CHARGE syndrome gastrointestinal involvement: From mouth to anus. Clin. Genet. 2017, 92, 10-17. [CrossRef]

45. Blake, K.D.; Prasad, C. CHARGE syndrome. Orphanet J. Rare Dis. 2006, 1, 34. [CrossRef]

46. Hale, C.L.; Niederriter, A.N.; Green, G.E.; Martin, D.M. Atypical phenotypes associated with pathogenic CHD7 variants and a proposal for broadening CHARGE syndrome clinical diagnostic criteria. Am. J. Med. Genet. Part A 2016, 170, 344-354. [CrossRef] [PubMed]

47. Trider, C.L.; Corsten, G.; Morrison, D.; Hefner, M.; Davenport, S.; Blake, K. Understanding obstructive sleep apnea in children with CHARGE syndrome. Int. J. Pediatr. Otorhinolaryngol. 2012, 76, 947-953. [CrossRef]

48. Blake, K.D.; Hartshorne, T.S.; Lawand, C.; Dailor, A.N.; Thelin, J.W. Cranial nerve manifestations in CHARGE syndrome. Am. J. Med. Genet. Part A 2008, 146, 585-592. [CrossRef] [PubMed]

49. Chang, J.H.; Park, D.H.; Shin, J.P.; Kim, I.T. Two cases of CHARGE syndrome with multiple congenital anomalies. Int. Ophthalmol. 2014, 34, 623-627. [CrossRef] [PubMed]

50. Devriendt, K.; Swillen, A.; Fryns, J.P. Deletion in chromosome region 22q11 in a child with CHARGE association. Clin. Genet. 1998, 53, 408-410. [CrossRef] [PubMed]

51. Brock, K.E.; Mathiason, M.A.; Rooney, B.L.; Williams, M.S. Quantitative analysis of limb anomalies in CHARGE syndrome: Correlation with diagnosis and characteristic CHARGE anomalies. Am. J. Med. Genet. Part A 2003, 123, 111-121. [CrossRef] [PubMed]

52. Ostrowski, P.J.; Zachariou, A.; Loveday, C.; Beleza-Meireles, A.; Bertoli, M.; Dean, J.; Douglas, A.G.L.; Ellis, I.; Foster, A.; Graham, J.M.; et al. The CHD8 overgrowth syndrome: A detailed evaluation of an emerging overgrowth phenotype in 27 patients. Am. J. Med. Genet. C Semin. Med. Genet. 2019, 181, 557-564. [CrossRef]

53. An, Y.; Zhang, L.; Liu, W.; Jiang, Y.; Chen, X.; Lan, X.; Li, G.; Hang, Q.; Wang, J.; Gusella, J.F.; et al. De novo variants in the Helicase-C domain of CHD8 are associated with severe phenotypes including autism, language disability and overgrowth. Hum. Genet. 2020, 139, 499-512. [CrossRef]

54. Douzgou, S.; Liang, H.W.; Metcalfe, K.; Somarathi, S.; Tischkowitz, M.; Mohamed, W.; Kini, U.; McKee, S.; Yates, L.; Bertoli, M.; et al. The clinical presentation caused by truncating CHD8 variants. Clin. Genet. 2019, 96, 72-84. [CrossRef] [PubMed]

55. Mefford, H.C.; Sharp, A.J.; Baker, C.; Itsara, A.; Jiang, Z.; Buysse, K.; Huang, S.; Maloney, V.K.; Crolla, J.A.; Baralle, D.; et al. Recurrent rearrangements of chromosome 1q21.1 and variable pediatric phenotypes. N. Engl. J. Med. 2008, 359, 1685-1699. [CrossRef]

56. Bernier, R.; Steinman, K.J.; Reilly, B.; Wallace, A.S.; Sherr, E.H.; Pojman, N.; Mefford, H.C.; Gerdts, J.; Earl, R.; Hanson, E.; et al. Clinical phenotype of the recurrent 1q21.1 copy-number variant. Genet. Med. 2016, 18, 341-349. [CrossRef]

57. Haldeman-Englert, C.R.; Jewett, T. 1q21.1 Recurrent Microdeletion; University of Washington, Seattle: Seattle, WA, USA, 2011.

58. Koivisto, M.; Akerblom, H.K.; Remes, M.; de La Chapelle, A. Primary hypothyroidism, growth hormone deficiency and congenital malformations in a child with the karyotype 46,XY,del(1)(q25q32). Acta Paediatr. Scand. 1976, 65, 513-518. [CrossRef] [PubMed]

59. Hu, P.; Wang, Y.; Meng, L.L.; Qin, L.; Ma, D.Y.; Yi, L.; Xu, Z.F. 1q25.2-q31.3 Deletion in a female with mental retardation, clinodactyly, minor facial anomalies but no growth retardation. Mol. Cytogenet. 2013, 6, 30. [CrossRef]

60. Kostopoulou, E.; Dastamani, A.; Caiulo, S.; Antell, H.; Flanagan, S.E.; Shah, P. Hyperinsulinaemic hypoglycaemia: A new presentation of 16p11.2 deletion syndrome. Clin. Endocrinol. 2019, 90, 766-769. [CrossRef] [PubMed]

61. Rein, B.; Yan, Z. 16p11.2 Copy Number Variations and Neurodevelopmental Disorders. Trends Neurosci. 2020, 43, 886-901. [CrossRef] 
62. Shiow, L.R.; Paris, K.; Akana, M.C.; Cyster, J.G.; Sorensen, R.U.; Puck, J.M. Severe combined immunodeficiency (SCID) and attention deficit hyperactivity disorder (ADHD) associated with a Coronin-1A mutation and a chromosome 16p11.2 deletion. Clin. Immunol. 2009, 131, 24-30. [CrossRef]

63. Balasubramanian, M.; Smith, K.; Mordekar, S.R.; Parker, M.J. Clinical report: AN INTERSTITIAL deletion of 16p13.11 detected by array CGH in a patient with infantile spasms. Eur. J. Med. Genet. 2011, 54, 314-318. [CrossRef]

64. Nagamani, S.C.; Erez, A.; Bader, P.; Lalani, S.R.; Scott, D.A.; Scaglia, F.; Plon, S.E.; Tsai, C.H.; Reimschisel, T.; Roeder, E.; et al. Phenotypic manifestations of copy number variation in chromosome 16p13.11. Eur. J. Hum. Genet. 2011, 19, 280-286. [CrossRef]

65. de Kovel, C.G.; Trucks, H.; Helbig, I.; Mefford, H.C.; Baker, C.; Leu, C.; Kluck, C.; Muhle, H.; von Spiczak, S.; Ostertag, P.; et al. Recurrent microdeletions at 15q11.2 and 16p13.11 predispose to idiopathic generalized epilepsies. Brain 2010, 133, 23-32. [CrossRef] [PubMed]

66. Landing, B.H.; Sugarman, G.; Dixon, L.G. Eccrine sweat gland anatomy in cockayne syndrome: A possible diagnostic aid. Pediatr. Pathol. 1983, 1, 349-353. [CrossRef] [PubMed]

67. Wilson, B.T.; Stark, Z.; Sutton, R.E.; Danda, S.; Ekbote, A.V.; Elsayed, S.M.; Gibson, L.; Goodship, J.A.; Jackson, A.P.; Keng, W.T.; et al. The Cockayne Syndrome Natural History (CoSyNH) study: Clinical findings in 102 individuals and recommendations for care. Genet. Med. 2016, 18, 483-493. [CrossRef] [PubMed]

68. Pasquier, L.; Laugel, V.; Lazaro, L.; Dollfus, H.; Journel, H.; Edery, P.; Goldenberg, A.; Martin, D.; Heron, D.; Le Merrer, M.; et al. Wide clinical variability among 13 new Cockayne syndrome cases confirmed by biochemical assays. Arch. Dis. Child 2006, 91, 178-182. [CrossRef]

69. Ovaert, C.; Cano, A.; Chabrol, B. Aortic dilatation in Cockayne syndrome. Am. J. Med. Genet. Part A 2007, 143, 2604-2606. [CrossRef] [PubMed]

70. Laugel, V. Cockayne Syndrome; University of Washington, Seattle: Seattle, WA, USA, 2000.

71. Speiser, P.W.; Arlt, W.; Auchus, R.J.; Baskin, L.S.; Conway, G.S.; Merke, D.P.; Meyer-Bahlburg, H.F.L.; Miller, W.L.; Murad, M.H.; Oberfield, S.E.; et al. Congenital Adrenal Hyperplasia Due to Steroid 21-Hydroxylase Deficiency: An Endocrine Society Clinical Practice Guideline. J. Clin. Endocrinol. Metab. 2018, 103, 4043-4088. [CrossRef]

72. Tamhane, S.; Rodriguez-Gutierrez, R.; Iqbal, A.M.; Prokop, L.J.; Bancos, I.; Speiser, P.W.; Murad, M.H. Cardiovascular and Metabolic Outcomes in Congenital Adrenal Hyperplasia: A Systematic Review and Meta-Analysis. J. Clin. Endocrinol. Metab. 2018, 103, 4097-4103. [CrossRef]

73. Deardorff, M.A.; Noon, S.E.; Krantz, I.D. Cornelia de Lange Syndrome; University of Washington, Seattle: Seattle, WA, USA, 2016.

74. Mariani, M.; Decimi, V.; Bettini, L.R.; Maitz, S.; Gervasini, C.; Masciadri, M.; Ajmone, P.; Kullman, G.; Dinelli, M.; Panceri, R.; et al. Adolescents and adults affected by Cornelia de Lange syndrome: A report of 73 Italian patients. Am. J. Med. Genet. C Semin. Med. Genet. 2016, 172, 206-213. [CrossRef]

75. Zambrelli, E.; Fossati, C.; Turner, K.; Taiana, M.; Vignoli, A.; Gervasini, C.; Russo, S.; Furia, F.; Masciadri, M.; Ajmone, P.; et al. Sleep disorders in Cornelia de Lange syndrome. Am. J. Med. Genet. C Semin. Med. Genet. 2016, 172, 214-221. [CrossRef]

76. Ichiyama, T.; Hayashi, T.; Tanaka, H.; Nishikawa, M.; Furukawa, S. Hearing impairment in two boys with Cornelia de Lange syndrome. Brain Dev. 1994, 16, 485-487. [CrossRef]

77. Decimi, V.; Parma, B.; Panceri, R.; Fossati, C.; Mariani, M.; Russo, S.; Gervasini, C.C.; Cheli, M.; Cereda, A.; Selicorni, A. Use of nutritional devices in Cornelia de Lange syndrome: Data from a large Italian cohort. Am. J. Med. Genet. Part A 2018, 176, 1865-1871. [CrossRef] [PubMed]

78. Gripp, K.W.; Rauen, R.K.A. Costello Syndrome; University of Washington, Seattle: Seattle, WA, USA, 2019. Available online: https: / / www.ncbi.nlm.nih.gov / books/NBK1507/ (accessed on 12 October 2020).

79. Cakir, M.; Arici, C.; Tacoy, S.; Karayalcin, U. A case of Costello with parathyroid adenoma and hyperprolactinemia. Am. J. Med. Genet. Part A 2004, 124, 196-199. [CrossRef] [PubMed]

80. Fujikawa, Y.; Sugai, K.; Fukumizu, M.; Hanaoka, S.; Sasaki, M.; Kaga, M. Three cases of Costello syndrome presenting with intractable epilepsy and profound psychomotor retardation/regression. No To Hattatsu 2001, 33, 430-435.

81. Cerruti Mainardi, P. Cri du Chat syndrome. Orphanet J. Rare Dis. 2006, 1, 33. [CrossRef] [PubMed]

82. Adams, D.J.; Clark, D.A. Common genetic and epigenetic syndromes. Pediatr. Clin. N. Am. 2015, 62, 411-426. [CrossRef]

83. Van Buggenhout, G.J.; Pijkels, E.; Holvoet, M.; Schaap, C.; Hamel, B.C.; Fryns, J.P. Cri du chat syndrome: Changing phenotype in older patients. Am. J. Med. Genet. 2000, 90, 203-215. [CrossRef]

84. Harvard, C.; Malenfant, P.; Koochek, M.; Creighton, S.; Mickelson, E.C.; Holden, J.J.; Lewis, M.E.; Rajcan-Separovic, E. A variant Cri du Chat phenotype and autism spectrum disorder in a subject with de novo cryptic microdeletions involving 5 p15.2 and 3p24.3-25 detected using whole genomic array CGH. Clin. Genet. 2005, 67, 341-351. [CrossRef]

85. Kuechler, A.; Willemsen, M.H.; Albrecht, B.; Bacino, C.A.; Bartholomew, D.W.; van Bokhoven, H.; van den Boogaard, M.J.; Bramswig, N.; Büttner, C.; Cremer, K.; et al. De novo mutations in beta-catenin (CTNNB1) appear to be a frequent cause of intellectual disability: Expanding the mutational and clinical spectrum. Hum. Genet. 2015, 134, 97-109. [CrossRef]

86. de Ligt, J.; Willemsen, M.H.; van Bon, B.W.; Kleefstra, T.; Yntema, H.G.; Kroes, T.; Vulto-van Silfhout, A.T.; Koolen, D.A.; de Vries, P.; Gilissen, C.; et al. Diagnostic exome sequencing in persons with severe intellectual disability. N. Engl. J. Med. 2012, 367, 1921-1929. [CrossRef] 
87. Verhoeven, W.M.A.; Egger, J.I.M.; Jongbloed, R.E.; van Putten, M.M.; de Bruin-van Zandwijk, M.; Zwemer, A.S.; Pfundt, R.; Willemsen, M.H. A de novo CTNNB1 Novel Splice Variant in an Adult Female with Severe Intellectual Disability. Int. Med. Case Rep. J. 2020, 13, 487-492. [CrossRef]

88. Menon, V.K.; Sorur, T.M.M.; Al Ghafri, K.A.; Shahin, M.M.H.E. Scoliosis in Dandy-Walker syndrome: A case report and review of literature. J. Spine Surg. 2017, 3, 702-706. [CrossRef] [PubMed]

89. Society for Maternal-Fetal Medicine (SMFM); Monteagudo, A. Dandy-Walker Malformation. Am. J. Obstet. Gynecol. 2020, 223, B38-B41. [CrossRef] [PubMed]

90. Johnston, P.C.; Donnelly, D.E.; Donnelly, D.K.; Morrison, P.J.; Hunter, S.J. DiGeorge syndrome presenting as late onset hypocalcaemia in adulthood. Ulster Med. J. 2008, 77, 201-202. [PubMed]

91. Kar, P.S.; Ogoe, B.; Poole, R.; Meeking, D. Di-George syndrome presenting with hypocalcaemia in adulthood: Two case reports and a review. J. Clin. Pathol. 2005, 58, 655-657. [CrossRef]

92. Cuneo, B.F.; Driscoll, D.A.; Gidding, S.S.; Langman, C.B. Evolution of latent hypoparathyroidism in familial 22q11 deletion syndrome. Am. J. Med. Genet. 1997, 69, 50-55. [CrossRef]

93. Bassett, A.S.; Chow, E.W.; Husted, J.; Weksberg, R.; Caluseriu, O.; Webb, G.D.; Gatzoulis, M.A. Clinical features of 78 adults with 22q11 Deletion Syndrome. Am. J. Med. Genet. Part A 2005, 138, 307-313. [CrossRef]

94. Brauner, R.; de Gonneville, A.L.H.; Kindermans, C.; Le Bidois, J.; Prieur, M.; Lyonnet, S.; Souberbielle, J.C. Parathyroid function and growth in 22q11.2 deletion syndrome. J. Pediatr. 2003, 142, 504-508. [CrossRef]

95. Giardino, G.; Cirillo, E.; Maio, F.; Gallo, V.; Esposito, T.; Naddei, R.; Grasso, F.; Pignata, C. Gastrointestinal involvement in patients affected with 22q11.2 deletion syndrome. Scand. J. Gastroenterol. 2014, 49, 274-279. [CrossRef]

96. Fagerberg, C.R.; Graakjaer, J.; Heinl, U.D.; Ousager, L.B.; Dreyer, I.; Kirchhoff, M.; Rasmussen, A.A.; Lautrup, C.K.; Birkebaek, N.; Sorensen, K. Heart defects and other features of the 22q11 distal deletion syndrome. Eur. J. Med. Genet. 2013, 56, 98-107. [CrossRef]

97. Evers, L.J.; van Amelsvoort, T.A.; Candel, M.J.; Boer, H.; Engelen, J.J.; Curfs, L.M. Psychopathology in adults with 22q11 deletion syndrome and moderate and severe intellectual disability. J. Intellect. Disabil. Res. 2014, 58, 915-925. [CrossRef]

98. Rauch, A.; Zink, S.; Zweier, C.; Thiel, C.T.; Koch, A.; Rauch, R.; Lascorz, J.; Hüffmeier, U.; Weyand, M.; Singer, H.; et al. Systematic assessment of atypical deletions reveals genotype-phenotype correlation in 22q11.2. J. Med. Genet. 2005, 42, 871-876. [CrossRef] [PubMed]

99. Couser, N.L.; Pande, C.K.; Walsh, J.M.; Tepperberg, J.; Aylsworth, A.S. Camptodactyly and the 22q11.2 deletion syndrome. Am. J. Med. Genet. Part A 2017, 173, 515-518. [CrossRef] [PubMed]

100. Eaton, C.B.; Thomas, R.H.; Hamandi, K.; Payne, G.C.; Kerr, M.P.; Linden, D.E.J.; Owen, M.J.; Cunningham, A.C.; Bartsch, U.; Struik, S.S.; et al. Epilepsy and seizures in young people with 22q11.2 deletion syndrome: Prevalence and links with other neurodevelopmental disorders. Epilepsia 2019, 60, 818-829. [CrossRef] [PubMed]

101. Schneider, M.; Debbané, M.; Bassett, A.S.; Chow, E.W.; Fung, W.L.; van den Bree, M.; Owen, M.; Murphy, K.C.; Niarchou, M.; Kates, W.R.; et al. Psychiatric disorders from childhood to adulthood in 22q11.2 deletion syndrome: Results from the International Consortium on Brain and Behavior in 22q11.2 Deletion Syndrome. Am. J. Psychiatry 2014, 171, 627-639. [CrossRef]

102. Fung, W.L.; Butcher, N.J.; Costain, G.; Andrade, D.M.; Boot, E.; Chow, E.W.; Chung, B.; Cytrynbaum, C.; Faghfoury, H.; Fishman, L.; et al. Practical guidelines for managing adults with 22q11.2 deletion syndrome. Genet. Med. 2015, 17, 599-609. [CrossRef]

103. Vorstman, J.A.; Breetvelt, E.J.; Thode, K.I.; Chow, E.W.; Bassett, A.S. Expression of autism spectrum and schizophrenia in patients with a 22q11.2 deletion. Schizophr. Res. 2013, 143, 55-59. [CrossRef]

104. Leader, G.; Murray, M.; O’Súilleabháin, P.S.; Maher, L.; Naughton, K.; Arndt, S.; White, K.; Traina, I.; Mannion, A. Relationship between parent-reported gastrointestinal symptoms, sleep problems, autism spectrum disorder symptoms, and behavior problems in children and adolescents with 22q11.2 deletion syndrome. Res. Dev. Disabil. 2020, 104, 103698. [CrossRef]

105. Shprintzen, R.J.; Goldberg, R.B.; Lewin, M.L.; Sidoti, E.J.; Berkman, M.D.; Argamaso, R.V.; Young, D. A new syndrome involving cleft palate, cardiac anomalies, typical facies, and learning disabilities: Velo-cardio-facial syndrome. Cleft. Palate J. 1978, $15,56-62$.

106. Witchel, S.F. Disorders of sex development. Best Pract. Res. Clin. Obstet. Gynaecol. 2018, 48, 90-102. [CrossRef]

107. Godfrey, L.M. Mental health outcomes among individuals with 46,XY disorders of sex development: A systematic review. J. Health Psychol. 2021, 26, 40-59. [CrossRef]

108. Grinspon, R.P.; Bedecarrás, P.; Ballerini, M.G.; Iñiguez, G.; Rocha, A.; Resende, E.A.M.R.; Brito, V.N.; Milani, C.; Figueroa Gacitúa, V.; Chiesa, A.; et al. Early onset of primary hypogonadism revealed by serum anti-Müllerian hormone determination during infancy and childhood in trisomy 21. Int. J. Androl. 2011, 34, e487-e498. [CrossRef] [PubMed]

109. Whooten, R.; Schmitt, J.; Schwartz, A. Endocrine manifestations of Down syndrome. Curr. Opin. Endocrinol. Diabetes Obes. 2018, 25, 61-66. [CrossRef] [PubMed]

110. Capone, G.T.; Chicoine, B.; Bulova, P.; Stephens, M.; Hart, S.; Crissman, B.; Videlefsky, A.; Myers, K.; Roizen, N.; Esbensen, A.; et al. Co-occurring medical conditions in adults with Down syndrome: A systematic review toward the development of health care guidelines. Am. J. Med. Genet. Part A 2018, 176, 116-133. [CrossRef] [PubMed]

111. Benhaourech, S.; Drighil, A.; Hammiri, A.E. Congenital heart disease and Down syndrome: Various aspects of a confirmed association. Cardiovasc. J. Afr. 2016, 27, 287-290. [CrossRef] [PubMed] 
112. Contestabile, A.; Benfenati, F.; Gasparini, L. Communication breaks-Down: From neurodevelopment defects to cognitive disabilities in Down syndrome. Prog. Neurobiol. 2010, 91, 1-22. [CrossRef] [PubMed]

113. Grieco, J.; Pulsifer, M.; Seligsohn, K.; Skotko, B.; Schwartz, A. Down syndrome: Cognitive and behavioral functioning across the lifespan. Am. J. Med. Genet. C Semin. Med. Genet. 2015, 169, 135-149. [CrossRef] [PubMed]

114. Thakker, S.; Persily, J.; Najari, B.B. Kallman syndrome and central non-obstructive azoospermia. Best Pract. Res. Clin. Endocrinol. Metab. 2020, 34, 101475. [CrossRef]

115. Dodé, C.; Hardelin, J.-P. Kallmann syndrome. Eur. J. Hum. Genet. 2009, 17, 139-146. [CrossRef]

116. Geng, D.; Zhang, H.; Liu, X.; Fei, J.; Jiang, Y.; Liu, R.; Wang, R.; Zhang, G. Identification of KISS1R gene mutations in disorders of non-obstructive azoospermia in the northeast population of China. J. Clin. Lab. Anal. 2020, 34, e23139. [CrossRef] [PubMed]

117. Moalla, M.; Kacem, F.H.; Al-Mutery, A.F.; Mahfood, M.; Mejdoub-Rekik, N.; Abid, M.; Mnif-Feki, M.; Hadj Kacem, H. Nonstop mutation in the Kisspeptin 1 receptor (KISS1R) gene causes normosmic congenital hypogonadotropic hypogonadism. J. Assist. Reprod. Genet. 2019, 36, 1273-1280. [CrossRef]

118. Pivnick, E.K.; Velagaleti, G.V.; Wilroy, R.S.; Smith, M.E.; Rose, S.R.; Tipton, R.E.; Tharapel, A.T. Jacobsen syndrome: Report of a patient with severe eye anomalies, growth hormone deficiency, and hypothyroidism associated with deletion 11 (q23q25) and review of 52 cases. J. Med. Genet. 1996, 33, 772-778. [CrossRef] [PubMed]

119. Mattina, T.; Perrotta, C.S.; Grossfeld, P. Jacobsen syndrome. Orphanet J. Rare Dis. 2009, 4, 9. [CrossRef] [PubMed]

120. Sirvent, N.; Monpoux, F.; Pedeutour, F.; Fraye, M.; Philip, P.; Ticchioni, M.; Turc-Carel, C.; Mariani, R.Jacobsen's syndrome, thrombopenia and humoral immunodeficiency. Arch. Pediatr. 1998, 5, 1338-1340. [CrossRef]

121. von Bubnoff, D.; Kreiss-Nachtsheim, M.; Novak, N.; Engels, E.; Engels, H.; Behrend, C.; Propping, P.; de la Salle, H.; Bieber, T. Primary immunodeficiency in combination with transverse upper limb defect and anal atresia in a 34-year-old patient with Jacobsen syndrome. Am. J. Med. Genet. Part A 2004, 126, 293-298. [CrossRef]

122. Bachmann-Gagescu, R.; Dempsey, J.C.; Bulgheroni, S.; Chen, M.L.; D’Arrigo, S.; Glass, I.A.; Heller, T.; Héon, E.; Hildebrandt, F.; Joshi, N.; et al. Healthcare recommendations for Joubert syndrome. Am. J. Med. Genet. Part A 2020, 182, 229-249. [CrossRef] [PubMed]

123. Fleming, L.R.; Doherty, D.A.; Parisi, M.A.; Glass, I.A.; Bryant, J.; Fischer, R.; Turkbey, B.; Choyke, P.; Daryanani, K.; Vemulapalli, M.; et al. Prospective Evaluation of Kidney Disease in Joubert Syndrome. Clin. J. Am. Soc. Nephrol. 2017, 12, 1962-1973. [CrossRef]

124. Hoeve, H.L.J.; Brooks, A.S.; Smit, L.S. JS-X syndrome: A multiple congenital malformation with vocal cord paralysis, ear deformity, hearing loss, shoulder musculature underdevelopment, and X-linked recessive inheritance. Int. J. Pediatr. Otorhinolaryngol. 2015, 79, 1164-1170. [CrossRef]

125. Wang, Y.R.; Xu, N.X.; Wang, J.; Wang, X.M. Kabuki syndrome: Review of the clinical features, diagnosis and epigenetic mechanisms. World J. Pediatr. 2019, 15, 528-535. [CrossRef]

126. Moon, J.E.; Lee, S.J.; Ko, C.W. A de novo KMT2D mutation in a girl with Kabuki syndrome associated with endocrine symptoms: A case report. BMC Med. Genet. 2018, 19, 102. [CrossRef]

127. Adam, M.P.; Hudgins, L.; Hannibal, M. Kabuki Syndrome; University of Washington, Seattle: Seattle, WA, USA, 2019. Available online: https: / / www.ncbi.nlm.nih.gov/books /NBK62111/ (accessed on 12 October 2020).

128. Philip, N.; Meinecke, P.; David, A.; Dean, J.; Ayme, S.; Clark, R.; Gross-Kieselstein, E.; Hosenfeld, D.; Moncla, A.; Muller, D. Kabuki make-up (Niikawa-Kuroki) syndrome: A study of 16 non-Japanese cases. Clin. Dysmorphol. 1992, 1, 63-77. [CrossRef]

129. Atalay, Y.O.; Kaya, C.; Ustun, Y.B.; Sahinoglu, A.H. Anesthesia management in a patient with kabuki syndrome. Med. Arch. 2014, 68, 359-360. [CrossRef] [PubMed]

130. Kennedy, J.; Goudie, D.; Blair, E.; Chandler, K.; Joss, S.; McKay, V.; Green, A.; Armstrong, R.; Lees, M.; Kamien, B.; et al. KAT6A Syndrome: Genotype-phenotype correlation in 76 patients with pathogenic KAT6A variants. Genet. Med. 2019, 21, 850-860. [CrossRef] [PubMed]

131. Urreizti, R.; Lopez-Martin, E.; Martinez-Monseny, A.; Pujadas, M.; Castilla-Vallmanya, L.; Pérez-Jurado, L.A.; Serrano, M.; Natera-de Benito, D.; Martínez-Delgado, B.; Posada-de-la-Paz, M.; et al. Five new cases of syndromic intellectual disability due to KAT6A mutations: Widening the molecular and clinical spectrum. Orphanet J. Rare Dis. 2020, 15, 44. [CrossRef] [PubMed]

132. Salzano, A.; D’Assante, R.; Heaney, L.M.; Monaco, F.; Rengo, G.; Valente, P.; Pasquali, D.; Bossone, E.; Gianfrilli, D.; Lenzi, A.; et al. Klinefelter syndrome, insulin resistance, metabolic syndrome, and diabetes: Review of literature and clinical perspectives. Endocrine 2018, 61, 194-203. [CrossRef]

133. Han, S.J.; Kim, K.S.; Kim, W.; Kim, J.H.; Lee, Y.H.; Nam, J.S.; Seo, J.A.; Kim, B.K.; Lee, J.; Chung, J.O.; et al. Obesity and Hyperglycemia in Korean Men with Klinefelter Syndrome: The Korean Endocrine Society Registry. Endocrinol. Metab. 2016, 31, 598-603. [CrossRef]

134. Ferlin, A.; Schipilliti, M.; Foresta, C. Bone density and risk of osteoporosis in Klinefelter syndrome. Acta Paediatr. 2011, 100, 878-884. [CrossRef]

135. Akcan, N.; Poyrazoğlu, Ş.; Baş, F.; Bundak, R.; Darendeliler, F. Klinefelter Syndrome in Childhood: Variability in Clinical and Molecular Findings. J. Clin. Res. Pediatr. Endocrinol. 2018, 10, 100-107. [CrossRef]

136. Salzano, A.; Arcopinto, M.; Marra, A.M.; Bobbio, E.; Esposito, D.; Accardo, G.; Giallauria, F.; Bossone, E.; Vigorito, C.; Lenzi, A.; et al. Klinefelter syndrome, cardiovascular system, and thromboembolic disease: Review of literature and clinical perspectives. Eur. J. Endocrinol. 2016, 175, R27-R40. [CrossRef] 
137. Beuers, U.; Richter, W.O.; Ritter, M.M.; Wiebecke, B.; Schwandt, P. Klinefelter's syndrome and liver adenoma. J. Clin. Gastroenterol. 1991, 13, 214-216. [CrossRef]

138. Fentiman, I.S. The endocrinology of male breast cancer. Endocr.-Relat. Cancer 2018, 25, R365-R373. [CrossRef]

139. Gregory, L.C.; Shah, P.; Sanner, J.R.F.; Arancibia, M.; Hurst, J.; Jones, W.D.; Spoudeas, H.; Le Quesne Stabej, P.; Williams, H.J.; Ocaka, L.A.; et al. Mutations in MAGEL2 and L1CAM Are Associated With Congenital Hypopituitarism and Arthrogryposis. J. Clin. Endocrinol. Metab. 2019, 104, 5737-5750. [CrossRef]

140. Isik, E.; Onay, H.; Atik, T.; Akgun, B.; Cogulu, O.; Ozkinay, F. Clinical and genetic features of L1 syndrome patients: Definition of two novel mutations. Clin. Neurol. Neurosurg. 2018, 172, 20-23. [CrossRef] [PubMed]

141. Stumpel, C.; Vos, Y.J. L1 Syndrome; University of Washington, Seattle: Seattle, WA, USA, 2004. Available online: https://www. ncbi.nlm.nih.gov / books /NBK1484/ (accessed on 12 October 2020).

142. Burglen, L.; Héron, D.; Moerman, A.; Dieux-Coeslier, A.; Bourguignon, J.P.; Bachy, A.; Carel, J.C.; Cormier-Daire, V.; Manouvrier, S.; Verloes, A. Myhre syndrome: New reports, review, and differential diagnosis. J. Med. Genet. 2003, 40, 546. [CrossRef]

143. García-Cruz, D.; Figuera, L.E.; Feria-Velazco, A.; Sánchez-Corona, J.; García-Cruz, M.O.; Ramírez-Duenãs, R.M.; HernandezCórdova, A.; Ruiz, M.X.; Bitar-Alatorre, W.E.; Ramírez-Dueñas, M.L. The Myhre syndrome: Report of two cases. Clin. Genet. 1993, 44, 203-207. [CrossRef] [PubMed]

144. Le Goff, C.; Michot, C.; Cormier-Daire, V. Myhre syndrome. Clin. Genet. 2014, 85, 503-513. [CrossRef] [PubMed]

145. Gutmann, D.H.; Ferner, R.E.; Listernick, R.H.; Korf, B.R.; Wolters, P.L.; Johnson, K.J. Neurofibromatosis type 1. Nat. Rev. Dis. Primers 2017, 3, 17004. [CrossRef] [PubMed]

146. Cohen, J.S.; Levy, H.P.; Sloan, J.; Dariotis, J.; Biesecker, B.B. Depression among adults with neurofibromatosis type 1: Prevalence and impact on quality of life. Clin. Genet. 2015, 88, 425-430. [CrossRef] [PubMed]

147. Sani, I.; Albanese, A. Endocrine Long-Term Follow-Up of Children with Neurofibromatosis Type 1 and Optic Pathway Glioma. Horm. Res. Paediatr. 2017, 87, 179-188. [CrossRef]

148. Favere, A.M.; Tsukumo, D.M.; Matos, P.S.; Santos, S.L.; Lalli, C.A. Association between atypical parathyroid adenoma and neurofibromatosis. Arch. Endocrinol. Metab. 2015, 59, 460-466. [CrossRef] [PubMed]

149. Fossali, E.; Signorini, E.; Intermite, R.C.; Casalini, E.; Lovaria, A.; Maninetti, M.M.; Rossi, L.N. Renovascular disease and hypertension in children with neurofibromatosis. Pediatr. Nephrol. 2000, 14, 806-810. [CrossRef]

150. Heervä, E.; Koffert, A.; Jokinen, E.; Kuorilehto, T.; Peltonen, S.; Aro, H.T.; Peltonen, J. A controlled register-based study of 460 neurofibromatosis 1 patients: Increased fracture risk in children and adults over 41 years of age. J. Bone Miner. Res. 2012, 27, 2333-2337. [CrossRef]

151. Kinori, M.; Hodgson, N.; Zeid, J.L. Ophthalmic manifestations in neurofibromatosis type 1. Surv. Ophthalmol. 2018, 63, 518-533. [CrossRef]

152. Lidzba, K.; Granström, S.; Lindenau, J.; Mautner, V.F. The adverse influence of attention-deficit disorder with or without hyperactivity on cognition in neurofibromatosis type 1. Dev. Med. Child Neurol. 2012, 54, 892-897. [CrossRef]

153. Leschziner, G.D.; Golding, J.F.; Ferner, R.E. Sleep disturbance as part of the neurofibromatosis type 1 phenotype in adults. Am. J. Med. Genet. Part A 2013, 161, 1319-1322. [CrossRef]

154. Terry, A.R.; Jordan, J.T.; Schwamm, L.; Plotkin, S.R. Increased Risk of Cerebrovascular Disease Among Patients With Neurofibromatosis Type 1: Population-Based Approach. Stroke 2016, 47, 60-65. [CrossRef]

155. Roberts, A.E.; Allanson, J.E.; Tartaglia, M.; Gelb, B.D. Noonan syndrome. Lancet 2013, 381, 333-342. [CrossRef]

156. Tartaglia, M.; Gelb, B.D.; Zenker, M. Noonan syndrome and clinically related disorders. Best Pract Res. Clin. Endocrinol. Metab. 2011, 25, 161-179. [CrossRef]

157. Johnston, J.J.; van der Smagt, J.J.; Rosenfeld, J.A.; Pagnamenta, A.T.; Alswaid, A.; Baker, E.H.; Blair, E.; Borck, G.; Brinkmann, J.; Craigen, W.; et al. Autosomal recessive Noonan syndrome associated with biallelic LZTR1 variants. Genet. Med. 2018, 20, 1175-1185. [CrossRef] [PubMed]

158. Synofzik, M.; Hufnagel, R.B.; Züchner, S. PNPLA6-Related Disorders; University of Washington, Seattle: Seattle, WA, USA, 2014. Available online: https:/ / www.ncbi.nlm.nih.gov/books/NBK247161/ (accessed on 12 October 2020).

159. Yehia, L.; Eng, C. PTEN Hamartoma Tumor Syndrome; University of Washington, Seattle: Seattle, WA, USA, 2001. Available online: https: / / www.ncbi.nlm.nih.gov / books /NBK1488/ (accessed on 12 October 2020).

160. Pal, A.; Barber, T.M.; Van de Bunt, M.; Rudge, S.A.; Zhang, Q.; Lachlan, K.L.; Cooper, N.S.; Linden, H.; Levy, J.C.; Wakelam, M.J.O.; et al. PTEN Mutations as a Cause of Constitutive Insulin Sensitivity and Obesity. N. Engl. J. Med. 2012, 367, 1002-1011. [CrossRef] [PubMed]

161. Yehia, L.; Eng, C. 65 YEARS OF THE DOUBLE HELIX: One gene, many endocrine and metabolic syndromes: PTEN-opathies and precision medicine. Endocr.-Relat. Cancer 2018, 25, T121-T140. [CrossRef] [PubMed]

162. Cassidy, S.B.; Schwartz, S.; Miller, J.L.; Driscoll, D.J. Prader-Willi syndrome. Genet. Med. 2012, 14, 10-26. [CrossRef] [PubMed]

163. Diene, G.; Mimoun, E.; Feigerlova, E.; Caula, S.; Molinas, C.; Grandjean, H.; Tauber, M.; PWS, F.R.C.f. Endocrine disorders in children with Prader-Willi syndrome-data from 142 children of the French database. Horm. Res. Paediatr. 2010, 74, 121-128. [CrossRef] [PubMed]

164. Rosenberg, A.G.W.; Pellikaan, K.; Poitou, C.; Goldstone, A.P.; Høybye, C.; Markovic, T.; Grugni, G.; Crinò, A.; Caixàs, A.; Coupaye, M.; et al. Central Adrenal Insufficiency Is Rare in Adults With Prader-Willi Syndrome. J. Clin. Endocrinol. Metab. 2020, 105, e2563-e2571. [CrossRef] [PubMed] 
165. l'Allemand, D.; Eiholzer, U.; Schlumpf, M.; Steinert, H.; Riesen, W. Cardiovascular risk factors improve during 3 years of growth hormone therapy in Prader-Willi syndrome. Eur. J. Pediatr. 2000, 159, 835-842. [CrossRef]

166. Purtell, L.; Viardot, A.; Campbell, L.V. Vitamin D levels in primary growth hormone deficiency disorder Prader-Willi syndrome. Endocrine 2016, 53, 619-620. [CrossRef] [PubMed]

167. Kuhlmann, L.; Joensson, I.M.; Froekjaer, J.B.; Krogh, K.; Farholt, S. A descriptive study of colorectal function in adults with Prader-Willi Syndrome: High prevalence of constipation. BMC Gastroenterol. 2014, 14, 63. [CrossRef] [PubMed]

168. Steinhausen, H.C.; Eiholzer, U.; Hauffa, B.P.; Malin, Z. Behavioural and emotional disturbances in people with Prader-Willi Syndrome. J. Intellect. Disabil. Res. 2004, 48, 47-52. [CrossRef]

169. Bantim, Y.C.V.; Kussaba, S.T.; de Carvalho, G.P.; Garcia-Junior, I.R.; Roman-Torres, C.V.G. Oral health in patients with Prader-Willi syndrome: Current perspectives. Clin. Cosmet. Investig. Dent. 2019, 11, 163-170. [CrossRef]

170. Kawano, H.; Ikeda, T.; Shimazaki, K.; Arakawa, S.; Matsumoto, Y.; Hayano, M.; Maemura, K. Successful treatment of heart failure in an adult patient with Prader-Willi syndrome. Intern. Med. 2013, 52, 771-776. [CrossRef]

171. Sinnema, M.; Boer, H.; Collin, P.; Maaskant, M.A.; van Roozendaal, K.E.; Schrander-Stumpel, C.T.; Curfs, L.M. Psychiatric illness in a cohort of adults with Prader-Willi syndrome. Res. Dev. Disabil. 2011, 32, 1729-1735. [CrossRef]

172. Percy, A.K.; Lane, J.B. Rett syndrome: Clinical and molecular update. Curr. Opin. Pediatr. 2004, 16, 670-677. [CrossRef] [PubMed]

173. Kaur, S.; Christodoulou, J. MECP2 Disorders; University of Washington, Seattle: Seattle, WA, USA, 2019. Available online: https://www.ncbi.nlm.nih.gov/books/NBK1497/ (accessed on 12 October 2020).

174. Gold, W.A.; Krishnarajy, R.; Ellaway, C.; Christodoulou, J. Rett Syndrome: A Genetic Update and Clinical Review Focusing on Comorbidities. ACS Chem. Neurosci. 2018, 9, 167-176. [CrossRef] [PubMed]

175. Kumar, A.; Jaryal, A.; Gulati, S.; Chakrabarty, B.; Singh, A.; Deepak, K.K.; Pandey, R.M.; Gupta, N.; Sapra, S.; Kabra, M.; et al. Cardiovascular Autonomic Dysfunction in Children and Adolescents With Rett Syndrome. Pediatr. Neurol. 2017, 70, 61-66. [CrossRef] [PubMed]

176. Killian, J.T.; Lane, J.B.; Lee, H.S.; Skinner, S.A.; Kaufmann, W.E.; Glaze, D.G.; Neul, J.L.; Percy, A.K. Scoliosis in Rett Syndrome: Progression, Comorbidities, and Predictors. Pediatr. Neurol. 2017, 70, 20-25. [CrossRef] [PubMed]

177. Specchio, N.; Carotenuto, A.; Trivisano, M.; Cappelletti, S.; Digilio, C.; Capolino, R.; Di Capua, M.; Fusco, L.; Vigevano, F. Ring 21 chromosome presenting with epilepsy and intellectual disability: Clinical report and review of the literature. Am. J. Med Genet. Part A 2011, 155, 911-914. [CrossRef]

178. Norman, M.; Wainstein, B.; Anazodo, A.; Turner, A.; Ma, C.; Payne, K.; Tangye, S.G.; Gray, P. Combined Immunodeficiency with Ring Chromosome 21. J. Clin. Immunol. 2018, 38, 251-256. [CrossRef]

179. Gallagher, E.R.; Ratisoontorn, C.; Cunningham, M.L. Saethre-Chotzen Syndrome; University of Washington, Seattle: Seattle, WA, USA, 2019. Available online: https:/ /www.ncbi.nlm.nih.gov/books/NBK1189/ (accessed on 12 October 2020).

180. Pelc, A.; Mikulewicz, M. Saethre-Chotzen syndrome: Case report and literature review. Dent. Med. Probl. 2018, 55, 217-225. [CrossRef]

181. Kilcoyne, S.; Luscombe, C.; Scully, P.; Jayamohan, J.; Magdum, S.; Wall, S.; Johnson, D.; Wilkie, A.O.M. Language Development, Hearing Loss, and Intracranial Hypertension in Children With TWIST1-Confirmed Saethre-Chotzen Syndrome. J. Craniofac. Surg. 2019, 30, 1506-1511. [CrossRef]

182. Campeau, P.M.; Lu, J.T.; Dawson, B.C.; Fokkema, I.F.; Robertson, S.P.; Gibbs, R.A.; Lee, B.H. The KAT6B-related disorders genitopatellar syndrome and Ohdo/SBBYS syndrome have distinct clinical features reflecting distinct molecular mechanisms. Hum. Mutat. 2012, 33, 1520-1525. [CrossRef]

183. Day, R.; Beckett, B.; Donnai, D.; Fryer, A.; Heidenblad, M.; Howard, P.; Kerr, B.; Mansour, S.; Maye, U.; McKee, S.; et al. A clinical and genetic study of the Say/Barber/Biesecker/Young-Simpson type of Ohdo syndrome. Clin. Genet. 2008, 74, 434-444. [CrossRef]

184. White, S.M.; Adès, L.C.; Amor, D.; Liebelt, J.; Bankier, A.; Baker, E.; Wilson, M.; Savarirayan, R. Two further cases of Ohdo syndrome delineate the phenotypic variability of the condition. Clin. Dysmorphol. 2003, 12, 109-113. [CrossRef] [PubMed]

185. Weiss, K.; Terhal, P.A.; Cohen, L.; Bruccoleri, M.; Irving, M.; Martinez, A.F.; Rosenfeld, J.A.; Machol, K.; Yang, Y.; Liu, P.; et al. De Novo Mutations in CHD4, an ATP-Dependent Chromatin Remodeler Gene, Cause an Intellectual Disability Syndrome with Distinctive Dysmorphisms. Am. J. Hum. Genet. 2016, 99, 934-941. [CrossRef] [PubMed]

186. Weiss, K.; Lazar, H.P.; Kurolap, A.; Martinez, A.F.; Paperna, T.; Cohen, L.; Smeland, M.F.; Whalen, S.; Heide, S.; Keren, B.; et al. The CHD4-related syndrome: A comprehensive investigation of the clinical spectrum, genotype-phenotype correlations, and molecular basis. Genet. Med. 2020, 22, 389-397. [CrossRef]

187. Anderson, J.; Viskochil, D.; O'Gorman, M.; Gonzales, C. Gastrointestinal complications of Russell-Silver syndrome: A pilot study. Am. J. Med. Genet. 2002, 113, 15-19. [CrossRef] [PubMed]

188. Marsaud, C.; Rossignol, S.; Tounian, P.; Netchine, I.; Dubern, B. Prevalence and management of gastrointestinal manifestations in Silver-Russell syndrome. Arch. Dis. Child 2015, 100, 353-358. [CrossRef] [PubMed]

189. Al Kaissi, A.; Ganger, R.; Mindler, G.; Karner, C.; Klaushofer, K.; Grill, F. Correction of the axial and appendicular deformities in a patient with Silver-Russel syndrome. Afr. J. Paediatr. Surg. 2015, 12, 36-40. [CrossRef] [PubMed]

190. Parker, M.J.; Deshpande, C.; Rankin, J.; Wilson, L.C.; Balasubramanian, M.; Hall, C.M.; Wagner, B.E.; Pollitt, R.; Dalton, A.; Bishop, N.J. Type 1 collagenopathy presenting with a Russell-Silver phenotype. Am. J. Med. Genet. Part A 2011, 155, 1414-1418. [CrossRef] 
191. Prasad, N.R.; Reddy, P.A.; Karthik, T.S.; Chakravarthy, M.; Ahmed, F. A rare case of Silver-Russell syndrome associated with growth hormone deficiency and urogenital abnormalities. Indian J. Endocrinol. Metab. 2012, 16, S307-S309. [CrossRef]

192. Donoghue, S.E.; Pitt, J.J.; Boneh, A.; White, S.M. Smith-Lemli-Opitz syndrome: Clinical and biochemical correlates. J. Pediatr. Endocrinol. Metab. 2018, 31, 451-459. [CrossRef]

193. Andersson, H.C.; Frentz, J.; Martínez, J.E.; Tuck-Muller, C.M.; Bellizaire, J. Adrenal insufficiency in Smith-Lemli-Opitz syndrome. Am. J. Med. Genet. 1999, 82, 382-384. [CrossRef]

194. Nowaczyk, M.J.; Wassif, C.A. Smith-Lemli-Opitz Syndrome; University of Washington, Seattle: Seattle, WA, USA, 2020. Available online: https://www.ncbi.nlm.nih.gov/books/NBK1143/ (accessed on 12 October 2020).

195. Tint, G.S.; Irons, M.; Elias, E.R.; Batta, A.K.; Frieden, R.; Chen, T.S.; Salen, G. Defective cholesterol biosynthesis associated with the Smith-Lemli-Opitz syndrome. N. Engl. J. Med. 1994, 330, 107-113. [CrossRef] [PubMed]

196. Tierney, E.; Nwokoro, N.A.; Porter, F.D.; Freund, L.S.; Ghuman, J.K.; Kelley, R.I. Behavior phenotype in the RSH/Smith-LemliOpitz syndrome. Am. J. Med. Genet. 2001, 98, 191-200. [CrossRef]

197. Shayota, B.J.; Elsea, S.H. Behavior and sleep disturbance in Smith-Magenis syndrome. Curr. Opin. Psychiatry 2019, 32, 73-78. [CrossRef] [PubMed]

198. Sotos, J.F.; Dodge, P.R.; Muirhead, D.; Crawford, J.D.; Talbot, N.B. Cerebral gigantism in childhood. a syndrome of excessively rapid growth and acromegalic features and a nonprogressive neurologic disorder. N. Engl. J. Med. 1964, 271, 109-116. [CrossRef] [PubMed]

199. Baujat, G.; Cormier-Daire, V. Sotos syndrome. Orphanet J. Rare Dis. 2007, 2, 36. [CrossRef] [PubMed]

200. Foster, A.; Zachariou, A.; Loveday, C.; Ashraf, T.; Blair, E.; Clayton-Smith, J.; Dorkins, H.; Fryer, A.; Gener, B.; Goudie, D.; et al. The phenotype of Sotos syndrome in adulthood: A review of 44 individuals. Am. J. Med. Genet. C Semin. Med. Genet. 2019, 181, 502-508. [CrossRef]

201. Goldstein, D.J.; Ward, R.E.; Moore, E.; Fremion, A.S.; Wappner, R.S. Overgrowth, congenital hypotonia, nystagmus, strabismus, and mental retardation: Variant of dominantly inherited Sotos sequence? Am. J. Med. Genet. 1988, 29, 783-792. [CrossRef]

202. Balci, T.B.; Strong, A.; Kalish, J.M.; Zackai, E.; Maris, J.M.; Reilly, A.; Surrey, L.F.; Wertheim, G.B.; Marcadier, J.L.; Graham, G.E.; et al. Tatton-Brown-Rahman syndrome: Six individuals with novel features. Am. J. Med. Genet. A 2020, 182, 673-680. [CrossRef]

203. Tatton-Brown, K.; Zachariou, A.; Loveday, C.; Renwick, A.; Mahamdallie, S.; Aksglaede, L.; Baralle, D.; Barge-Schaapveld, D.; Blyth, M.; Bouma, M.; et al. The Tatton-Brown-Rahman Syndrome: A clinical study of 55 individuals with de novo constitutive DNMT3A variants. Wellcome Open Res. 2018, 3, 46. [CrossRef]

204. Heinen, C.A.; Losekoot, M.; Sun, Y.; Watson, P.J.; Fairall, L.; Joustra, S.D.; Zwaveling-Soonawala, N.; Oostdijk, W.; van den Akker, E.L.; Alders, M.; et al. Mutations in TBL1X Are Associated With Central Hypothyroidism. J. Clin. Endocrinol. Metab. 2016, 101, 4564-4573. [CrossRef]

205. García, M.; Barreda-Bonis, A.C.; Jiménez, P.; Rabanal, I.; Ortiz, A.; Vallespín, E.; Del Pozo, Á.; Martínez-San Millán, J.; González-Casado, I.; Moreno, J.C. Central Hypothyroidism and Novel Clinical Phenotypes in Hemizygous Truncation of TBL1X. J. Endocr. Soc. 2019, 3, 119-128. [CrossRef] [PubMed]

206. Chung, R.H.; Ma, D.; Wang, K.; Hedges, D.J.; Jaworski, J.M.; Gilbert, J.R.; Cuccaro, M.L.; Wright, H.H.; Abramson, R.K.; Konidari, I.; et al. An X chromosome-wide association study in autism families identifies TBL1X as a novel autism spectrum disorder candidate gene in males. Mol. Autism. 2011, 2, 18. [CrossRef] [PubMed]

207. Kara, C.; Üstyol, A.; Yılmaz, A.; Altundağ, E.; Oğur, G. Premature ovarian failure due to tetrasomy X in an adolescent girl. Eur. J. Pediatr. 2014, 173, 1627-1630. [CrossRef] [PubMed]

208. Uppal, S.; Jee, Y.H.; Lightbourne, M.; Han, J.C.; Stratakis, C.A. Combined pituitary hormone deficiency in a girl with 48 , XXXX and Rathke's cleft cyst. Hormones 2017, 16, 92-98. [CrossRef]

209. Alvaro-Gracia, J.M.; Humbria, A.; García-Vicuña, R.; Ariza, A.; García-Vadillo, A.; Laffón, A. Systemic lupus erythematosus and tetrasomy-X. J. Rheumatol. 1989, 16, 1486-1488.

210. Slae, M.; Heshin-Bekenstein, M.; Simckes, A.; Heimer, G.; Engelhard, D.; Eisenstein, E.M. Female polysomy-X and systemic lupus erythematosus. Semin. Arthritis Rheum. 2014, 43, 508-512. [CrossRef]

211. Linden, M.G.; Bender, B.G.; Robinson, A. Sex chromosome tetrasomy and pentasomy. Pediatrics 1995, 96, 672-682.

212. Jayaraman, D.; Carvalho, K.S.; Hasbani, D.M. A case report of hypersomnia in tetrasomy X improved with medical therapy. Clin. Case Rep. 2018, 6, 893-895. [CrossRef]

213. Tartaglia, N.R.; Howell, S.; Sutherland, A.; Wilson, R.; Wilson, L. A review of trisomy X (47,XXX). Orphanet J. Rare Dis. 2010, 5, 8. [CrossRef]

214. Linden, M.G.; Bender, B.G.; Harmon, R.J.; Mrazek, D.A.; Robinson, A. 47,XXX: What is the prognosis? Pediatrics 1988, 82, 619-630. [CrossRef]

215. McCray, B.A.; Schindler, A.; Hoover-Fong, J.E.; Sumner, C.J. Autosomal Dominant TRPV4 Disorders; University of Washington, Seattle: Seattle, WA, USA, 2014. Available online: https:/ / www.ncbi.nlm.nih.gov/books/NBK201366/ (accessed on 12 October 2020).

216. Henske, E.P.; Jóźwiak, S.; Kingswood, J.C.; Sampson, J.R.; Thiele, E.A. Tuberous sclerosis complex. Nat. Rev. Dis. Primers 2016, 2, 16035. [CrossRef]

217. Jabir, S.; Al-Hyassat, S. Histological diagnosis of cardiac lipoma in an adult with tuberous sclerosis. BMJ Case Rep. 2013. [CrossRef] [PubMed] 
218. Gravholt, C.H.; Andersen, N.H.; Conway, G.S.; Dekkers, O.M.; Geffner, M.E.; Klein, K.O.; Lin, A.E.; Mauras, N.; Quigley, C.A.; Rubin, K.; et al. Clinical practice guidelines for the care of girls and women with Turner syndrome: Proceedings from the 2016 Cincinnati International Turner Syndrome Meeting. Eur. J. Endocrinol. 2017, 177, G1-G70. [CrossRef]

219. De Groote, K.; Demulier, L.; De Backer, J.; De Wolf, D.; De Schepper, J.; T'sjoen, G.; De Backer, T. Arterial hypertension in Turner syndrome: A review of the literature and a practical approach for diagnosis and treatment. J. Hypertens. 2015, 33, $1342-1351$. [CrossRef] [PubMed]

220. Twite, M.D.; Stenquist, S.; Ing, R.J. Williams syndrome. Paediatr. Anaesth. 2019, 29, 483-490. [CrossRef] [PubMed]

221. Nordstrøm, M.; Paus, B.; Andersen, L.F.; Kolset, S.O. Dietary aspects related to health and obesity in Williams syndrome, Down syndrome, and Prader-Willi syndrome. Food Nutr. Res. 2015, 59, 25487. [CrossRef]

222. Güven, A. Seven cases with Williams-Beuren syndrome: Endocrine evaluation and long-term follow-up. J. Pediatr. Endocrinol. Metab. 2017, 30, 159-165. [CrossRef]

223. Nicita, F.; Garone, G.; Spalice, A.; Savasta, S.; Striano, P.; Pantaleoni, C.; Spartà, M.V.; Kluger, G.; Capovilla, G.; Pruna, D.; et al. Epilepsy is a possible feature in Williams-Beuren syndrome patients harboring typical deletions of the $7 \mathrm{q} 11.23 \mathrm{critical}$ region. Am. J. Med. Genet. Part A 2016, 170, 148-155. [CrossRef]

224. Huang, Y.C.; Lee, C.T.; Wu, M.Z.; Liu, S.Y.; Tung, Y.C.; Ho, H.N.; Tsai, W.Y. The spectrum of 45,X/46,XY mosaicism in Taiwanese children: The experience of a single center. J. Formos. Med. Assoc. 2019, 118, 450-456. [CrossRef]

225. Telvi, L.; Lebbar, A.; Del Pino, O.; Barbet, J.P.; Chaussain, J.L. 45,X/46,XY mosaicism: Report of 27 cases. Pediatrics 1999, 104, 304-308. [CrossRef]

226. Tartaglia, N.; Ayari, N.; Howell, S.; D'Epagnier, C.; Zeitler, P. 48,XXYY, 48,XXXY and 49,XXXXY syndromes: Not just variants of Klinefelter syndrome. Acta Paediatr. 2011, 100, 851-860. [CrossRef]

227. Blumling, A.A.; Martyn, K.; Talboy, A.; Close, S. Rare sex chromosome variation 48,XXYY: An integrative review. Am. J. Med. Genet. C Semin. Med. Genet. 2020, 184, 386-403. [CrossRef]

228. Tartaglia, N.; Davis, S.; Hench, A.; Nimishakavi, S.; Beauregard, R.; Reynolds, A.; Fenton, L.; Albrecht, L.; Ross, J.; Visootsak, J.; et al. A new look at XXYY syndrome: Medical and psychological features. Am. J. Med. Genet. A 2008, 146, 1509-1522. [CrossRef] [PubMed]

229. Tartaglia, N.R.; Wilson, R.; Miller, J.S.; Rafalko, J.; Cordeiro, L.; Davis, S.; Hessl, D.; Ross, J. Autism Spectrum Disorder in Males with Sex Chromosome Aneuploidy: XXY/Klinefelter Syndrome, XYY, and XXYY. J. Dev. Behav. Pediatr. $2017,38,197-207$. [CrossRef] [PubMed]

230. Krauser, D.G.; Lloyd-Jones, D.M.; Chae, C.U.; Cameron, R.; Anwaruddin, S.; Baggish, A.L.; Chen, A.; Tung, R.; Januzzi, J.L., Jr. Effect of body mass index on natriuretic peptide levels in patients with acute congestive heart failure: A ProBNP Investigation of Dyspnea in the Emergency Department (PRIDE) substudy. Am. Heart J. 2005, 149, 744-750. [CrossRef] [PubMed]

231. Nederland, Z. Farmacotherapeutisch Kompas. Available online: https:/ / farmacotherapeutischkompas.nl (accessed on 12 October 2020).

232. Mervis, C.B.; Velleman, S.L. Children with Williams Syndrome: Language, Cognitive, and Behavioral Characteristics and their Implications for Intervention. Perspect. Lang. Learn. Educ. 2011, 18, 98-107. [CrossRef]

233. van't Leven, M.; Zielhuis, G.A.; van der Meer, J.W.; Verbeek, A.L.; Bleijenberg, G. Fatigue and chronic fatigue syndrome-like complaints in the general population. Eur. J. Public Health 2009, 20, 251-257. [CrossRef]

234. Drossman, D.A.; Li, Z.; Andruzzi, E.; Temple, R.D.; Talley, N.J.; Thompson, W.G.; Whitehead, W.E.; Janssens, J.; Funch-Jensen, P.; Corazziari, E.; et al. U.S. householder survey of functional gastrointestinal disorders. Prevalence, sociodemography, and health impact. Dig. Dis. Sci. 1993, 38, 1569-1580. [CrossRef]

235. Centraal Bureau Voor de Statistiek. Gezondheid en Zorggebruik; Persoonskenmerken. 2021. Available online: https://opendata. cbs.nl/statline/\#/CBS/nl/dataset/83005NED/table?dl=4486F (accessed on 11 March 2021).

236. Lionti, T.; Reid, S.M.; White, S.M.; Rowell, M.M. A population-based profile of 160 Australians with Prader-Willi syndrome: Trends in diagnosis, birth prevalence and birth characteristics. Am. J. Med. Genet. Part A 2015, 167, 371-378. [CrossRef]

237. Bar, C.; Diene, G.; Molinas, C.; Bieth, E.; Casper, C.; Tauber, M. Early diagnosis and care is achieved but should be improved in infants with Prader-Willi syndrome. Orphanet J. Rare Dis. 2017, 12, 118. [CrossRef]

238. López-Bastida, J.; Linertová, R.; Oliva-Moreno, J.; Posada-de-la-Paz, M.; Serrano-Aguilar, P.; Kanavos, P.; Taruscio, D.; Schieppati, A.; Iskrov, G.; Baji, P.; et al. Social/economic costs and health-related quality of life in patients with Prader-Willi syndrome in Europe. Eur. J. Health Econ. 2016, 17 (Suppl. 1), 99-108. [CrossRef] [PubMed]

239. Lopez-Bastida, J.; Oliva-Moreno, J.; Linertova, R.; Serrano-Aguilar, P. Social/economic costs and health-related quality of life in patients with rare diseases in Europe. Eur. J. Health Econ. 2016, 17 (Suppl. 1), 1-5. [CrossRef] [PubMed]

240. Chevreul, K.; Berg Brigham, K.; Clement, M.C.; Poitou, C.; Tauber, M.; Members of the BURQOL-RD Research Network listed in the Online Appendix. Economic burden and health-related quality of life associated with Prader-Willi syndrome in France. J. Intellect. Disabil. Res. 2016, 60, 879-890. [CrossRef] [PubMed]

241. Wijeysundera, H.C.; Machado, M.; Wang, X.; Van Der Velde, G.; Sikich, N.; Witteman, W.; Tu, J.V.; Lee, D.S.; Goodman, S.G.; Petrella, R.; et al. Cost-effectiveness of specialized multidisciplinary heart failure clinics in Ontario, Canada. Value Health 2010, 13, 915-921. [CrossRef]

242. Komenda, P.; Levin, A. Analysis of cardiovascular disease and kidney outcomes in multidisciplinary chronic kidney disease clinics: Complex disease requires complex care models. Curr. Opin. Nephrol. Hypertens. 2006, 15, 61-66. [CrossRef] 
243. Wallace, R.A. Clinical audit of gastrointestinal conditions occurring among adults with Down syndrome attending a specialist clinic. J. Intellect. Dev. Disabil. 2007, 32, 45-50. [CrossRef]

244. Real de Asua, D.; Quero, M.; Moldenhauer, F.; Suarez, C. Clinical profile and main comorbidities of Spanish adults with Down syndrome. Eur. J. Intern. Med. 2015, 26, 385-391. [CrossRef]

245. Farquhar, M.; Jacobson, M.; Braun, C.; Wolfman, W.; Kelly, C.; Allen, L.M.; Lega, I.C. Medical and gynecological comorbidities in adult women with Turner syndrome: Our multidisciplinary clinic experience. Climacteric 2019, 23, 32-37. [CrossRef]

246. Freriks, K.; Timmermans, J.; Beerendonk, C.C.; Verhaak, C.M.; Netea-Maier, R.T.; Otten, B.J.; Braat, D.D.; Smeets, D.F.; Kunst, D.H.; Hermus, A.R.; et al. Standardized multidisciplinary evaluation yields significant previously undiagnosed morbidity in adult women with Turner syndrome. J. Clin. Endocrinol. Metab. 2011, 96, E1517-E1526. [CrossRef]

247. Kahlert, E.; Blaschke, M.; Brockmann, K.; Freiberg, C.; Janssen, O.E.; Stahnke, N.; Strik, D.; Merkel, M.; Mann, A.; Liesenkötter, K.P.; et al. Deficient knowledge in adult Turner syndrome care as an incentive to found Turner centers in Germany. Endocr. Connect. 2019, 8, 1483-1492. [CrossRef]

248. Vincent, A.J.; Nguyen, H.H.; Ranasinha, S.; Vollenhoven, B. Increased detection of co-morbidities with evaluation at a dedicated adult Turner syndrome clinic. Climacteric 2017, 20, 442-447. [CrossRef] [PubMed]

249. Penninx, B.; Lange, S.M.M. Metabolic syndrome in psychiatric patients: Overview, mechanisms, and implications. Dialogues. Clin. Neurosci. 2018, 20, 63-73. [PubMed]

250. Steenbergen, H.A.; Van der Schans, C.P.; Van Wijck, R.; De Jong, J.; Waninge, A. Lifestyle Approaches for People With Intellectual Disabilities: A Systematic Multiple Case Analysis. J. Am. Med. Dir. Assoc. 2017, 18, 980-987.e3. [CrossRef] [PubMed]

251. Willems, M.; Waninge, A.; Hilgenkamp, T.I.M.; van Empelen, P.; Krijnen, W.P.; van der Schans, C.P.; Melville, C.A. Effects of lifestyle change interventions for people with intellectual disabilities: Systematic review and meta-analysis of randomized controlled trials. J. Appl. Res. Intellect. Disabil. 2018, 31, 949-961. [CrossRef] 